Article

\title{
Phenolome of Asian Agrimony Tea (Agrimonia asiatica Juz., Rosaceae): LC-MS Profile, $\alpha$-Glucosidase Inhibitory Potential and Stability
}

\author{
Nina I. Kashchenko ${ }^{1,2, *(1)}$ and Daniil N. Olennikov ${ }^{1}[$ \\ 1 Laboratory of Medical and Biological Research, Institute of General and Experimental Biology, Siberian \\ Division, Russian Academy of Science, 6 Sakh'yanovoy Street, Ulan-Ude 670047, Russia; \\ olennikovdn@mail.ru \\ 2 Department of Biology, Institute of Natural Sciences, North-Eastern Federal University, 58 Belinsky Street, \\ Yakutsk 677027, Russia \\ * Correspondence: ninkk@mail.ru; Tel.: +79-8342-17-340
}

Received: 21 August 2020; Accepted: 21 September 2020; Published: 23 September 2020

\begin{abstract}
Functional beverages constitute the rapidly increasing part of the functional food section and represent an area with a wide range of products including herbal-based beverages. We carried out screening investigations of the extracts of 85 Rosaceous tea plants. Among the extracts analyzed Agrimonia asiatica herb extract demonstrated the highest inhibitory activity against the enzyme $\alpha$-glucosidase $(20.29 \mu \mathrm{g} / \mathrm{mL})$. As a result of chromato-mass-spectrometric profiling of A. asiatica herb with high-performance liquid chromatography with photodiode array and electrospray triple quadrupole mass-spectrometric detection (HPLC-PDA-ESI-tQ-MS) 60 compounds were identified, including catechins, ellagitannins, flavones, flavonols, gallotannins, hydroxycinnamates, procyanidins, most for the very first time. The analysis of the seasonal variation of metabolites in A. asiatica herb demonstrated that the phenolic content was highest in summer samples and lower in spring and autumn. HPLC activity-based profiling was utilized to identify compounds of $A$. asiatica herb with the maximal $\alpha$-glucosidase inhibitory activity. The most pronounced inhibition of $\alpha$-glucosidase was observed for agrimoniin, while less significant results of inhibition were revealed for ellagic acid and isoquercitrin. The evaluation of phenolic content in A. asiatica herbal teas with the subsequent determination of $\alpha$-glucosidase inhibiting potential was discovered. Maximum inhibition of $\alpha$-glucosidase was observed for hot infusion $(75.33 \mu \mathrm{g} / \mathrm{mL}$ ) and the minimum for $30 \mathrm{~min}$ decoction $(159.14 \mu \mathrm{g} / \mathrm{mL})$. Our study demonstrated that $A$. asiatica herbal tea is a prospective functional beverage in which dietary intake may help to reduce blood glucose.
\end{abstract}

Keywords: Agrimonia asiatica; Rosaceae; ellagitannins; agrimoniin; high-performance liquid chromatography; mass spectrometry; $\alpha$-glucosidase inhibition

\section{Introduction}

Over the past decade, there has been increasing interest in the manufacturing and consumption of functional foods because they ensure considerable health benefits, such as declining the risk of chronic diseases and enhancement of physiological conditions in the human body. In view of the era of pandemics, consumers are interested in functional foods that strengthen the immune system. A recent survey related to the coronavirus disease (COVID) pandemic found that one in five consumers cited immune system support as the top reason for the acquisition of functional foods [1]. However, the accessibility of bioactive ingredients in functional foods may become a problem, as the demand for these products can rise dramatically. Thus, the search for new functional products supporting human health is essential for modern consumers. 
Various emerging technologies used in the modern food industry to produce new functional products with health-promoting effects as radio-frequency drying, electro-osmotic dewatering, ultrafiltration, etc. [2,3]. The functional beverage industries present the biggest and rapidly increasing part of the functional food sector comprising of food, beverages, and supplement segments [4]. There are various uses of functional beverages like milk-based drinks, probiotic beverages, energy drinks, caffeinated beverages, sports drinks, herbal-based functional beverages [5]. Herbal beverages (well-known as teas) have gained popularity among consumers who are concerned about their health. They have entered the developing niche market alongside other popular beverages such as tea or coffee which are also made using plant-based materials [6]. The interest of functional beverages has led to the introduction of a number of new herbal teas on the market intended to address particular health problems. For example, a few various types of teas have been reported to reduce blood glucose levels and have antidiabetic effects [7-10].

Diabetes mellitus (DM) is the most abundant chronic and metabolic illness characterized by an elevation in blood glucose levels because of the absolute or relative insulin deficit. The chronic complications of diabetes are broadly divided into microvascular (neuropathy, nephropathy, and retinopathy) and macrovascular (cardiovascular disease, stroke, peripheral artery disease) [11]. In accordance with the International Diabetes Federation, 327 million people at present suffer from $\mathrm{DM}$, and this figure will reach 438 million by 2045. Type 2 diabetes is the most abundant form of this disturbance, accounting for $90 \%$ of the total affected population [12]. Diabetes can be managed using a variety of strategies. Correct treatment demands control in preprandial and postprandial hyperglycemia.

The enzyme $\alpha$-glucosidase catalyzes the hydrolysis of the $\alpha$-glycosidic bond of oligosaccharides to release the monosaccharide units from food sources $[13,14]$. The inhibition of $\alpha$-glucosidase can delay the release of glucose of oligosaccharides and disaccharides from dietary complex carbohydrates and cause a decrease in postprandial hyperglycemia [15]. Acarbose and other hypoglycemic synthetic $\alpha$-glucosidase inhibitors have been endorsed for clinical use in the management of DM type 2 . Nevertheless, the use of similar medicinal agents is accompanied by undesirable gastrointestinal side-effects (flatulence, stomach ache, diarrhea) and may lead to a limitation in the duration of therapy [16]. So, novel treatment strategies are needed for the prevention or treatment of diabetes mellitus and recently herbal medicines have received the attention of researchers in this area. There is growing evidence that polyphenolic substances exhibit strong $\alpha$-glucosidase inhibitory activity and are responsible for the reduction of blood glucose levels [17,18]. Among plant polyphenols with antidiabetic properties, ellagitannins have attracted a high degree of interest. These compounds involve structurally complex plant phenolics that are widespread in higher plants, especially in the Rosaceae family, and can reach high molecular masses [19,20].

Although herbal products are generally considered safe due to their natural origin, they are a complex mixture of different substances for which detailed chemical profiling demands serious research [21]. As a part of our continued investigation of antidiabetic plant constituents of Rosaceous plants [22-25], the present work aimed to perform chromato-mass-spectrometric profiling of A. asiatica herb with high-performance liquid chromatography with photodiode array and electrospray triple quadrupole mass-spectrometric detection (HPLC-PDA-ESI-tQ-MS), as well as analysis of A. asiatica herb for inhibitory activity against digestive enzyme $\alpha$-glucosidase. This multisectoral approach is an important basis for promoting the bioactive compounds of $A$. asiatica herb for use in future antidiabetic drugs.

\section{Materials and Methods}

\subsection{Chemicals}

The following chemicals were acquired from Aktin Chemicals Inc. (Chengdu, China): kaempferol-3-O-(6"-p-coumaroyl)-glucoside (56, tiliroside); Chemwill Asia Co., Ltd. (Beijing, China): agrimoniin (36); ChemFaces (Wuhan, China): apigenin 7-O-(6"-O-malonylglucoside) (59); Extrasynthese 
(Lyon, France): 5-O-caffeoyl quinic acid (14, neochlorogenic acid); kaempferol-3-O-glucoside (49); kaempferol-3-O-rhamnoside (54); luteolin-7-O-glucoside (43, cynaroside); procyanidin B3 (12); quercetin-3-O-glucoside (41, isoquercitrin); quercetin-3-O-rhamnoside (48); Sigma-Aldrich (St. Louis, MO, USA): acarbose; apigenin-7-O-glucoside (50, cosmosiin); apigenin-7-O-glucuronide (51); bovine serum albumin (BSA); 4-O-caffeoylquinic acid (8); catechin (13); 1,3-di-O-caffeoylquinic acid (26); 3,5-di-O-caffeoylquinic acid (46); ellagic acid (38); epicatechin (23); epicatechin gallate (37); epigallocatechin (15); 1-O-galloyl-glucose (2); kaempferol-3-O-(6"-O-rhamnosyl)-glucoside (47, nicotiflorin); luteolin-7-O-glucuronide (44); procyanidin B1 (10); procyanidin B2 (16); quercetin-3-O-glucuronide (42); quercetin-3-O-(6"-malonyl)-glucoside (45); di-sodium hydrogen phosphate; $\alpha$-glucosidase from Saccharomyces cerevisiae (type 1, $10 \mathrm{U} / \mathrm{mg}$ ); lithium perchlorate; 4-nitrophenyl- $\alpha$-D-glucopyranoside; perchloric acid; potassium dihydrogen phosphate; sodium carbonate. 2-Pyrone-4,6-dicarboxylic acid (6), pedunculagin (7), and potentillin (32) were isolated previously from $C$. palustre [22]; tellimagrandins $(\mathbf{1 1}, \mathbf{1 9}, \mathbf{3 1}, \mathbf{4 0})$ were isolated earlier from Filipendula ulmaria [23]; gemin A (34), agrimonic acid A (33), agrimonic acid B (35) were isolated from Potentilla anserina [24], 6-hydroxyluteolin-7-O-glucoside (28) was isolated from Rhaponticum uniflorum [26].

\subsection{Plant Material}

Plant samples were collected in various Siberian regions (Table S1) and A. asiatica was collected in 2018 in the Zabaykalsky Krai, Aga (Agin-Buryat Okrug, 51 ${ }^{\circ} 12^{\prime} 4.55^{\prime \prime} \mathrm{N}, 115^{\circ} 9^{\prime} 56.86^{\prime \prime}$ E, $685 \mathrm{~m}$ ). For a more accurate study of the seasonal fluctuations of phenolic compounds in herb, the samples from three different stages of development were collected in May, July, and September. Individual sampling dates were May 15, July 16, and September 22. All samples were collected between 9 and 11 a.m. The herb samples were sealed in polyethylene bags in the field and placed into a cooler with ice. Upon return to the laboratory, they were air-dried for 10 days at room temperature in a ventilated fume cupboard to a moisture content of $9-12 \%$. Herb samples were stored at $4{ }^{\circ} \mathrm{C}$ in the Plant Repository (Institute of General and Experimental Biology). Then 3 obtained samples of the herb of each month were pooled together, resulting in 3 total samples of the seasonal collection. Voucher specimens of A. asiatica herb were No. ARo/ag-0911-31/0519 (May samples), ARo/ag-0911-32/0719 (July samples), ARo/ag-0911-32/0919 (September samples). Then the samples were ground in an A11 basic analytical mill (IKA ${ }^{\circledR}$-WerkeGmbh \& Co. KG, Staufen, Germany). Subsequently, the samples were sieved up to an average particle diameter of $0.5 \mathrm{~mm}$ applying sieving machine ERL-M1 (Zernotekhnika, Moscow, Russia).

\subsection{Plant Extracts Preparation}

To prepare the total extracts of 85 Rosaceous tea plants (powdered samples of herbs $(100 \mathrm{~g})$ of Agrimonia asiatica Juz., A. pilosa Ledeb., Alchemilla anisopoda Juz., A. flavescens Buser, A. subcrenata Buser, Armeniaca sibirica (L.) Lam., Chamaerhodos erecta (L.) Bunge, C. grandiflora (Pall. ex Schult.) Bunge, Cotoneaster lucidus Schltdl., Cotoneaster melanocarpus Fisch. ex Blytt, C. mongolicus Pojark., C. neopopovii Czerep., C. tjuliniae Pojark. ex Peschkova, C. uniflorus Bunge, Crataegus dahurica Koehne ex C.K.Schneid., C. maximowiczii C.K.Schneid., C. sanguinea Pall., Dasiphora fruticosa (L.) Rydb., Dryas grandis Juz., D. incisa Juz., D. integrifolia subsp. crenulata (Juz.) Kozhevn., D. oxyodonta Juz., D. sumneviczii Serg., Dasiphora parvifolia (Fisch.) Juz., Fragaria vesca L., F. orientalis Losinsk., Geum aleppicum Jacq., G. rivale L., Malus baccata (L.) Borkh., Padus avium Mill., Potentilla acaulis L., P. acervata Soják, P. adenotricha Vodop., P. anserina L., P. arenosa (Turcz.) Juz., P. argentea L., P. asperrima Turcz., P. biflora Willd. ex Schltdl., P. chrysantha Trevir., P. conferta Bunge, P. crantzii (Crantz) Beck ex Fritsch, P. desertorum Bunge, P. elegans Cham. \& Schltdl., P. evestita Th.Wolf, P. flagellaris Willd. ex Schltdl., P. fragarioides L., P. kryloviana Th.Wolf, P. leucophylla Pall., P. longifolia Willd. ex Schltdl., P. multifida L., P. mujensis Kurbatski, P. nivea L., P. norvegica L., P. nudicaulis Willd. ex Schltdl., P. ozjorensis Peschkova, P. sanguisorba Willd. ex Schltdl., P. saposhnikovii Kurbatski, P. sericea L., P. stipularis L., P. tanacetifolia Willd. ex Schltdl., P. tergemina Soják, P. verticillaris Stephan ex Willd., Prunus pedunculata (Pall.) Maxim., Rosa acicularis Lindl., R. davurica 
Pall., Rubus arcticus L., R. chamaemorus L., R. humulifolius C.A. Mey., R. matsumuranus H. Lev. \& Vaniot, R. saxatilis L., Sanguisorba alpina Bunge, S. officinalis L., Sibbaldia adpressa Bunge, S. procumbens L., Sibbaldianthe bifurca (L.) Kurtto and T.Erikss., S. bifurca subsp. orientalis (Juz.) Kurtto \& T.Erikss., Sorbaria pallasii (G. Don fil.) Pojark., S. sorbifolia (L.) A. Braun, Sorbus sibirica Hedl., Spiraea alpina Pall., S. aquilegifolia Pall., S. dahurica (Rupr.) Maxim., S. flexuosa Fisch. ex Cambess., S. media Schmidt, S. salicifolia L.) were extracted three times in glass conical flasks (2 L) with water (1 L) with stirring. The extracts have been sonicated for $60 \mathrm{~min}\left(50^{\circ} \mathrm{C}, 100 \mathrm{~W}, 35 \mathrm{kHz}\right)$. The extracts obtained were filtrated through a cellulose filter. Consequently, the extracts obtained were combined and then evaporated in vacuo until dryness. The obtained extracts were kept at $4{ }^{\circ} \mathrm{C}$ until further chemical investigations and analysis of biological activity.

2.4. Identification of Phenolic Compounds by High-Performance Liquid Chromatography with Photodiode Array Detection and Electrospray Ionization Triple Quadrupole Mass Spectrometric Detection

(HPLC-PDA-ESI-tQ-MS)

The phenolic compounds profiling was realized by reversed-phase high-performance liquid chromatography with photodiode array detection and electrospray ionization mass spectrometry (HPLC-PDA-ESI-tQ-MS) assay using liquid chromatograph LC-20 Prominence coupled with photodiode array detector SPD-M30A (wavelength range 200-600 nm), triple-quadrupole mass spectrometer LCMS 8050 (all Shimadzu, Columbia, MD, USA) and Mastro C18 column $(150 \times 2.1 \mathrm{~mm}$, $3 \mu \mathrm{m}$; Shimadzu GLC, Kyoto, Japan) at the column temperature $30^{\circ} \mathrm{C}$. The following eluents were used: A ( $0.4 \%$ formic acid in water) and B ( $0.4 \%$ formic acid in acetonitrile). The injection volume was $1 \mu \mathrm{L}$, and elution flow rate was $80 \mu \mathrm{L} / \mathrm{min}$. Gradient program: $0.0-2.0 \mathrm{~min} 5.0-7.5 \% \mathrm{~B}, 2.0-7.0 \mathrm{~min}$ $7.5-15.0 \%$ B, 7.0-11.0 $\min 15.0-38.0 \%$ B, $11.0-14.0 \mathrm{~min} 38.0-42.0 \% \mathrm{~B}, 14.0-20.0 \mathrm{~min} 42.0-80.0 \% \mathrm{~B}$, $20.0-25.0 \mathrm{~min} 80.0-100.0 \% \mathrm{~B}, 25.0-35.0 \mathrm{~min} 100.0-5.0 \%$ B. Mass spectrometric detection was used in negative ESI mode ( $-3 \mathrm{kV}$ source voltage, range of $m / z$ 100-1900, collision energy $5-40 \mathrm{eV})$. There were following temperature levels of ESI interface $\left(300^{\circ} \mathrm{C}\right)$, desolvation line $\left(250^{\circ} \mathrm{C}\right)$, and heat block $\left(400{ }^{\circ} \mathrm{C}\right)$. There were following flow rates of nebulizing gas $\left(\mathrm{N}_{2}, 3 \mathrm{~L} / \mathrm{min}\right)$, heating gas (air, $10 \mathrm{~L} / \mathrm{min}$ ), collision-induced dissociation gas ( $\mathrm{Ar}, 0.3 \mathrm{~mL} / \mathrm{min})$. The raw data were MS/MS acquired and processed with LabSolutions workstation software (Shimadzu) with the internal LC-MS library. The MS/MS detection parameters of the 44 compounds are shown in Table S2. The identification of compounds was done by the analysis of their retention time, ultraviolet, and mass-spectrometric data (MS, MS/MS) comparing the same parameters with the reference samples and/or literature data.

\subsection{HPLC-MS Quantification}

Quantification of compounds 1-60 were carried out using HPLC-MS data (full scan MS, peak area). The quantifications were realized under the conditions specified in Section 2.4. For the preparation of the stock solutions, 44 reference compounds $(2,6-16,19,23,26,28,31-51,53-56,58-60)$ were carefully weighed $(10 \mathrm{mg})$, then separately dissolved in methanol in a $\mathrm{W}$ flask $(10 \mathrm{~mL})$. An external standard calibration curve was constructed with the use of six data points $(100,50,25,10,5$, and $1 \mu \mathrm{g} / \mathrm{mL})$. The calibration curves were constructed by plotting the MS peak area vs. concentration levels. Correlation coefficients, $r^{2}$; standard deviation, $S_{\mathrm{XX}}$; limits of detection, LOD; limits of quantification, LOQ; linear ranges were computed applying early reference [27]. All analyses were performed in triplicate, data obtained were expressed as mean value \pm standard deviation (SD). To prepare a sample solution, $40 \mathrm{mg}$ of the crushed plant was placed in an Eppendorf tube, subsequently, $1 \mathrm{~mL}$ of $60 \%$ methanol was added. Thereafter the sample was subjected to ultrasonic extraction for $30 \mathrm{~min}$ at $50{ }^{\circ} \mathrm{C}$. The weight of the tube was reduced to an initial sign after cooling. For analysis, the extract obtained was filtered through $0.22 \mu \mathrm{m}$ polytetrafluoroethylene (PTFE) syringe filter and then injected into the HPLC system. 


\section{6. $\alpha$-Glucosidase Inhibitory Activity}

\subsubsection{Plant Extracts Analysis}

The $\alpha$-glucosidase inhibiting potential of plant extracts (Section 2.3) was carried out using spectrophotometric assay [28]. Enzyme $\alpha$-glucosidase was dissolved in phosphate buffer (PBS, pH 6.8) containing $0.2 \%$ BSA up to $0.5 \mathrm{U} / \mathrm{mL}$ concentration. Sample solution $(10 \mu \mathrm{L})$ in PBS at various concentrations up 10 to $1000 \mu \mathrm{g} / \mathrm{mL}$ was preblended with $490 \mu \mathrm{L}$ of PBS and $250 \mu \mathrm{L} p$-nitrophenyl $\alpha$-D-glucopyranoside $(5 \mathrm{mM})$ with subsequent $5 \mathrm{~min}$ preincubation $\left(37^{\circ} \mathrm{C}\right)$. Then $250 \mu \mathrm{L} \alpha$-glucosidase $(0.4 \mathrm{U} / \mathrm{mL})$ was added and incubated for $15 \mathrm{~min}\left(37^{\circ} \mathrm{C}\right) .2000 \mu \mathrm{L} \mathrm{Na}_{2} \mathrm{CO}_{3}(200 \mathrm{mM})$ was added. The absorbance was measured at $400 \mathrm{~nm}$ and calculated as described earlier [28]. Acarbose was applied as a positive control (the half maximal inhibitory concentration $\mathrm{IC}_{50} 1214.25 \pm 24.48 \mu \mathrm{g} / \mathrm{mL}$ ) while water was a negative control.

\subsubsection{HPLC Based Bioactivity Guided Profiling}

Aliquots $(100 \mu \mathrm{L})$ of $A$. asiatica extract solution $(10 \mathrm{mg} / \mathrm{mL})$ were separated under analytical HPLC conditions as described in Section 2.4. The collection of eluates $(40 \mu \mathrm{L})$ performed every $30 \mathrm{~s}$ in 96-well plates. After drying and redissolving the eluates in $10 \mu \mathrm{L}$ of PBS the eluates were investigated as described earlier [29]. $\alpha$-Glucosidase was dissolved in PBS which contained BSA (0.2\%) up to $0.5 \mathrm{U} / \mathrm{mL}$ concentration and then added $125 \mu \mathrm{L}$ of PBS and $60 \mu \mathrm{L}$ p-nitrophenyl- $\alpha$-D-glucopyranoside $(5 \mathrm{mM})$. The samples were incubated at $37^{\circ} \mathrm{C}$ for $5 \mathrm{~min}$ and then $60 \mu \mathrm{L} \alpha$-glucosidase $(0.4 \mathrm{U} / \mathrm{mL})$ was added. The samples were incubated at $37^{\circ} \mathrm{C}$ for $15 \mathrm{~min}$. Then $50 \mu \mathrm{L}$ sodium carbonate $(200 \mathrm{mM})$ was added. Absorbance (A400) was determined at $400 \mathrm{~nm}$. The rates (1-A400) were carried out to create HPLC-based bioactivity profiles of $A$. asiatica extract.

\subsection{Preparation of A. asiatica Herb Infusions and Decoctions}

To prepare the infusion of $A$. asiatica, accurately weighed herb $(1 \mathrm{~g})$ was mixed with water $(20,50$, 80 , or $100{ }^{\circ} \mathrm{C}$ ) in a conical flask and agitated $(40 \mathrm{~min})$, followed by the cooling at $20^{\circ} \mathrm{C}$ (if required), filtering throw a PTFE filter $(0.45 \mu \mathrm{m})$ into a volumetric flask $(100 \mathrm{~mL})$ and reducing the final volume with distilled water. The decoctions of A. asiatica were produced from accurately weighed herb $(1 \mathrm{~g})$ after mixing with distilled water $(100 \mathrm{~mL})$ in a conical flask, heating on a hotplate, and boiling ( 15 or $30 \mathrm{~min})$. The resultant decoctions were passed throw a PTFE filter $(0.45 \mu \mathrm{m})$ into a volumetric flask $(100 \mathrm{~mL})$ and the volume was filled with distilled water.

\subsection{Statistical Analysis}

Statistical analyses were carried out with the usage of ANOVA (one-way analysis of variance). The significance of the mean difference was established by Duncan's multiple range test. Differences were regarded as statistically considerable at $p<0.05$. The results are presented as mean values \pm standard deviations (SD) for three to five replicates.

\section{Results and Discussion}

\section{1. $\alpha$-Glucosidase Inhibiting Activity and Ellagitannins Content in 85 Rosaceous Tea Plants}

At the preliminary stage of investigation, we decided to screen the most common tea species of the Rosaceae family growing in Siberia to reveal the most active species with the highest $\alpha$-glucosidase inhibiting activity. The extracts of 85 Rosaceous tea plants were obtained and the content of ellagitannins was determined (Table 1). The maximal ellagitannin content was observed in Agrimonia asiatica extract $(63.61 \mathrm{mg} / \mathrm{g}$ ). The high content of ellagitannins (more than $40 \mathrm{mg} / \mathrm{g}$ ) has also been demonstrated for Geum aleppicum, Sibbaldianthe bifurca, Rosa acicularis, Rubus humilifolius, Chamaerhodos grandiflora species. Further, the study of $\alpha$-glucosidase inhibitory activity of the extracts investigated was carried out. We decided to divide the species investigated according to their inhibitory activity on $\alpha$-glucosidase 
into three groups: active ( $\mathrm{IC}_{50}<50 \mu \mathrm{g} / \mathrm{mL}$ ), medium ( $\mathrm{IC}_{50} 50-100 \mu \mathrm{g} / \mathrm{mL}$ ), and low active/inactive $\left(\mathrm{IC}_{50}>100 \mu \mathrm{g} / \mathrm{mL}\right)$. Based on this criterion, extracts with maximal content of ellagitannins significantly inhibited $\alpha$-glucosidase and were included in the active group.

Table 1. $\alpha$-Glucosidase inhibiting activity $\left(\mathrm{IC}_{50}, \mu \mathrm{g} / \mathrm{mL} \pm \text { S.D. }\right)^{*}$ and ellagitannin content $(\mathrm{ET}, \mathrm{mg} / \mathrm{g}$ of dry extract weight \pm S.D.) for extracts from 85 Rosaceous tea plants.

\begin{tabular}{|c|c|c|c|}
\hline Species & $\mathrm{IC}_{50}, \mu \mathrm{g} / \mathrm{mL}$ & $\mathrm{ET}, \mathrm{mg} / \mathrm{g}$ & Group \\
\hline Agrimonia asiatica & $20.29 \pm 0.42$ & $63.61 \pm 1.59$ & \multirow{6}{*}{ Active $\left(\mathrm{IC}_{50}<50 \mu \mathrm{g} / \mathrm{mL}\right)$} \\
\hline Geum aleppicum & $29.31 \pm 0.44$ & $52.03 \pm 1.43$ & \\
\hline Sibbaldianthe bifurca & $31.16 \pm 0.50$ & $51.45 \pm 1.28$ & \\
\hline Rosaacicularis & $32.33 \pm 0.54$ & $50.04 \pm 1.12$ & \\
\hline Rubus humulifolius & $33.63 \pm 0.71$ & $47.05 \pm 0.99$ & \\
\hline Chamaerhodos grandiflora & $39.26 \pm 0.70$ & $41.94 \pm 0.83$ & \\
\hline Waldstenia ternata & $52.18 \pm 1.04$ & $32.14 \pm 0.64$ & \multirow{19}{*}{ Medium $\left(\mathrm{IC}_{50}\right.$ 50-100 ㅆg/mL) } \\
\hline Potentilla conferta & $54.37 \pm 1.02$ & tr. & \\
\hline Rubus matsumuranus & $55.12 \pm 1.12$ & $34.16 \pm 0.71$ & \\
\hline Dasiphora fruticosa & $55.45 \pm 1.27$ & $22.18 \pm 0.51$ & \\
\hline Dryas oxyodonta & $58.48 \pm 1.28$ & tr. & \\
\hline Potentilla tergemina & $66.71 \pm 1.41$ & $6.30 \pm 0.12$ & \\
\hline Agrimonia pilosa & $67.82 \pm 1.36$ & $34.27 \pm 0.82$ & \\
\hline Dasiphora parvifolia & $68.93 \pm 1.57$ & $18.45 \pm 0.47$ & \\
\hline Potentilla longifolia & $68.86 \pm 1.51$ & tr. & \\
\hline Potentilla stipularis & $68.46 \pm 1.51$ & $19.57 \pm 0.45$ & \\
\hline Sanguisorba officinalis & $77.88 \pm 1.75$ & $14.85 \pm 0.31$ & \\
\hline Potentilla fragarioides & $79.03 \pm 1.58$ & $6.34 \pm 0.11$ & \\
\hline Crataegus sanguinea & $84.36 \pm 1.94$ & tr. & \\
\hline Cotoneaster melanocarpus & $90.12 \pm 2.16$ & tr. & \\
\hline Potentilla nudicaulis & $91.52 \pm 2.21$ & tr. & \\
\hline Sibbaldia procumbens & $92.61 \pm 2.31$ & $5.22 \pm 0.10$ & \\
\hline Fragaria vesca & $95.47 \pm 1.90$ & $7.36 \pm 0.14$ & \\
\hline Sanguisorba alpina & $96.07 \pm 2.49$ & $7.55 \pm 0.11$ & \\
\hline Potentilla tanacetifolia & $96.93 \pm 2.52$ & tr. & \\
\hline 60 Rosaceous species ${ }^{b}$ & $>100$ & tr. & Low active / inactive $\left(\mathrm{IC}_{50}>100 \mu \mathrm{g} / \mathrm{mL}\right)$ \\
\hline
\end{tabular}

* Acarbose was used as a reference compound $\left(\mathrm{IC}_{50} 1214.25 \pm 24.48 \mu \mathrm{g} / \mathrm{mL}\right) .{ }^{\mathrm{b}}$ Listed in Table S1. tr. - traces $(<0.01 \mathrm{mg} / \mathrm{g})$.

As a result of enzymatic analysis, it was revealed that $A$. asiatica extract inhibited $\alpha$-glucosidase with the highest $\mathrm{IC}_{50}$ value $(20.29 \mu \mathrm{g} / \mathrm{mL})$. Acarbose inhibited $\alpha$-glucosidase with $\mathrm{IC}_{50}$ value $1214.25 \mu \mathrm{g} / \mathrm{mL}$. To reveal the links among the ellagitannins content and biological activity, linear regression analysis was applied (Figure 1). A strong and positive correlation was observed between ellagitannins content and enzyme inhibiting activity $(\mathrm{r}=0.8612)$. The findings indicated that ellagitannins contributed to the inhibition of the enzyme $\alpha$-glucosidase. Based on the data obtained we decided to choose A. asiatica as the herb for further investigation. In addition, there is information about the antidiabetic activity of the genus Agrimonia in the literature [30,31]. The chemical composition of this plant species is insufficiently studied. Only the detection of ursolic acid in A. asiatica herb has been studied to this point [32]. To characterize the chemical composition of the genus Agrimonia, we compiled a review on the content of chemical compounds in representatives of this genus. According to the literature data, the known information about the chemical composition of Agrimonia species relates to A. pilosa and A. eupatoria in general. Thus, the presence of phenolic acids, resorcinols, phloroglucinols, hydroxycinnamates, coumarins, chromones, flavones, flavonols, flavanonols, catechins, proanthocyanidins, ellagitannins, sterols, triterpenoids, etc. were established (Table 2). 


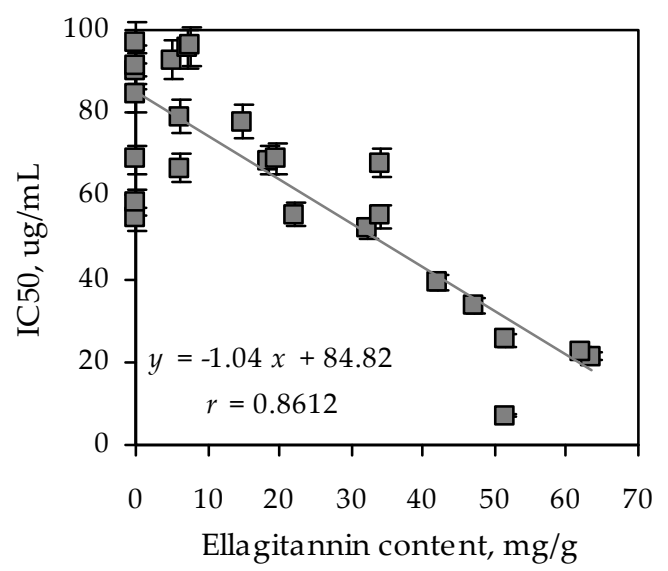

Figure 1. Correlation graph between ellagitannin content $(\mathrm{mg} / \mathrm{g}$; independent variable $x$ in regression equation) in extracts of Rosaceous species and their $\alpha$-glucosidase inhibiting activity $\left(\mathrm{IC}_{50}, \mu \mathrm{g} / \mathrm{mL}\right.$; dependent variable $y$ in regression equation). $r$-correlation coefficient.

Table 2. Known compounds found in Agrimonia species (literature data).

\begin{tabular}{|c|c|c|c|}
\hline \multirow{2}{*}{ Compound } & \multirow{2}{*}{ Species } & \multicolumn{2}{|c|}{ Found in [ref.] } \\
\hline & & Herb & Roots \\
\hline \multicolumn{4}{|l|}{ Phenolic Acids } \\
\hline & A. pilosa & [33] & \\
\hline \multirow[t]{2}{*}{ Protocatechuic acid } & A. eupatoria & [34] & \\
\hline & A. pilosa & [33] & \\
\hline $\begin{array}{l}\text { Isovanillic acid } \\
\text { Gallic acid }\end{array}$ & A. pilosa & [33] & \\
\hline \multirow[t]{2}{*}{ Gallic acid } & A. eupatoria & [35] & \\
\hline & A. pilosa & [33] & \\
\hline \multirow[t]{2}{*}{ Ellagic acid } & A. eupatoria & [36] & \\
\hline & A. pilosa & [37] & [38] \\
\hline Ellagic acid-4-O-Xyl $p$ & A. pilosa & & {$[38]$} \\
\hline \multicolumn{4}{|l|}{ Resorcinols } \\
\hline Agrimopiloside A & A. pilosa & [39] & \\
\hline Agrimopiloside B & A. pilosa & [39] & \\
\hline 1-Butanoyl-3,5-dimethyl-phloroglucinyl-6-O-Glcp Phloroglucinols & A. pilosa & [40] & \\
\hline Agrimol A & A. pilosa & & [41] \\
\hline Agrimol B & A. pilosa & [33] & [41] \\
\hline Agrimol C & A. pilosa & & [42] \\
\hline Agrimol D & A. pilosa & [33] & [41] \\
\hline Agrimol F & A. pilosa & & [42] \\
\hline Agrimol G & A. pilosa & & [42] \\
\hline Agrimophol & A. pilosa & & [41] \\
\hline Agripinol A & A. pilosa & [43] & \\
\hline Agripinol B & A. pilosa & [43] & \\
\hline Agripinol C & A. pilosa & [43] & \\
\hline \multirow{2}{*}{$\begin{array}{l}\text { Pseudoaspidin } \\
\alpha \text {-Cosin }\end{array}$} & A. pilosa & [43] & [44] \\
\hline & A. pilosa & [43] & \\
\hline \multirow{3}{*}{$p$-Coumaric acid } & & & \\
\hline & A. eupatoria & [34] & \\
\hline & A. pilosa & [33] & \\
\hline \multirow[t]{2}{*}{ 3-O-p-Coumaroylquinic acid } & A. eupatoria & [45] & \\
\hline & A. procera & [36] & \\
\hline \multirow[t]{2}{*}{ 1-O-Caffeoylquinic acid } & A. eupatoria & [36] & \\
\hline & A. procera & [36] & \\
\hline \multirow[t]{2}{*}{ 3-O-Caffeoylquinic acid } & A. eupatoria & [36] & \\
\hline & A. procera & [36] & \\
\hline 4-O-Caffeoylquinic acid & A. eupatoria & [36] & \\
\hline & A. procera & [36] & \\
\hline 5-O-Caffeoylquinic acid & A. eupatoria & [45] & \\
\hline & A. procera & [36] & \\
\hline
\end{tabular}


Table 2. Cont.

\begin{tabular}{|c|c|c|c|}
\hline \multirow{2}{*}{ Compound } & \multirow{2}{*}{ Species } & \multicolumn{2}{|c|}{ Found in [ref.] } \\
\hline & & Herb & Roots \\
\hline \multicolumn{4}{|l|}{ Coumarins } \\
\hline Agrimonolide & A. pilosa & {$[40]$} & [46] \\
\hline Agrimonolide-6-O-Glcp & A. pilosa & [47] & [48] \\
\hline (3S)-Agrimonolide-6-O-(6'-O-Araf)-Glcp & A. pilosa & [49] & \\
\hline Desmethylagrimonolide-6-O-Glcp & A. pilosa & [50] & \\
\hline (3S)-Desmethylagrimonolide-4'-O-Glc $p$ & A. pilosa & [49] & \\
\hline$(3 S, 4 R)-4$-Hydroxyagrimonolide-6-O-Glcp & A. pilosa & [49] & \\
\hline 5,7-Dihydroxy-2-propylchromone-7-O-Glcp Chromones & A. pilosa & [50] & \\
\hline Takanechromone C & A. pilosa & [50] & \\
\hline Petiolin E & A. pilosa & [33] & \\
\hline \multicolumn{4}{|l|}{ Flavones } \\
\hline Apigenin & A. eupatoria & [51] & \\
\hline \multirow[t]{3}{*}{ Apigenin-7-O-Glcp (= cosmosiin) } & A. eupatoria & [51] & \\
\hline & A. pilosa & [52] & \\
\hline & A. procera & [36] & \\
\hline \multirow[t]{3}{*}{ Apigenin-7-O-GlcA $p$} & A. eupatoria & [45] & \\
\hline & A. pilosa & [53] & \\
\hline & A. procera & [36] & \\
\hline Apigenin-7-O-(6"-Me)-GlcAp & A. pilosa & [54] & \\
\hline Apigenin-6-C-Glc $p$ (= isovitexin) & A. eupatoria & [34] & \\
\hline Apigenin-8-C-Glcp (= vitexin) & A. eupatoria & [45] & \\
\hline Luteolin & A. eupatoria & [51] & \\
\hline \multirow[t]{3}{*}{ Luteolin-7-O-Glc $p$ (= cynaroside) } & A. eupatoria & [51] & \\
\hline & A. pilosa & [52] & \\
\hline & A. procera & [36] & \\
\hline \multirow[t]{3}{*}{ Luteolin-7-O-GlcA $p$} & A. eupatoria & [45] & \\
\hline & A. pilosa & [53] & \\
\hline & A. procera & [36] & \\
\hline Luteolin-7-O-(6"-Me)-GlcAp & A. pilosa & [37] & \\
\hline Luteolin-7-O-(6"-But)-GlcAp & A. pilosa & [37] & \\
\hline Luteolin-3'-O-Glcp & A. pilosa & [37] & \\
\hline \multirow[t]{2}{*}{ Pinocembrin } & A. pilosa & [33] & \\
\hline & & & \\
\hline \multirow[t]{2}{*}{ Kaempferol } & A. eupatoria & [55] & \\
\hline & A. pilosa & [56] & \\
\hline Kaempferol-3-O-Arap & A. pilosa & [33] & \\
\hline \multirow[t]{2}{*}{ Kaempferol-3-O-Rhap (= afzelin) } & A. eupatoria & [55] & \\
\hline & A. pilosa & [56] & \\
\hline \multirow[t]{3}{*}{ Kaempferol-3-O-Glc $p$ (= astragalin) } & A. eupatoria & [55] & \\
\hline & A. pilosa & [56] & \\
\hline & A. procera & [36] & \\
\hline Kaempferol-3-O-(6"-Ac)-Glcp & A. pilosa & [33] & \\
\hline Kaempferol-3-O-[6'-O-(S)-3-hydroxy-3-methylglutaryl]-Glcp & A. pilosa & [37] & \\
\hline \multirow[t]{2}{*}{ Kaempferol-3-O-(6"-p-CouA)-Glcp (= tiliroside) } & A. eupatoria & [34] & \\
\hline & A. pilosa & [57] & \\
\hline Kaempferol-7-O-Glcp & A. pilosa & [37] & \\
\hline Kaempferol-7-O-GlcAp & A. pilosa & [37] & \\
\hline Kaempferol-3-O-Rut (= nicotiflorin) & A. eupatoria & [55] & \\
\hline Kaempferide & A. eupatoria & [55] & \\
\hline Kaempferide-3-O-Rhap & A. eupatoria & [55] & \\
\hline Quercetin & A. eupatoria & [51] & \\
\hline & A. pilosa & [58] & \\
\hline Quercetin-3-O-Glcp (= isoquercitrin) & A. eupatoria & [36] & \\
\hline & A. procera & [36] & \\
\hline Quercetin-3-O-(6"-O-Gall)-Glc $p$ & A. eupatoria & [36] & \\
\hline & A. procera & [36] & \\
\hline Quercetin-3-O-Gal $p$ (= hyperoside) & A. eupatoria & [34] & \\
\hline & A. pilosa & [58] & \\
\hline & A. procera & [36] & \\
\hline Quercetin-3-O-Rhap (= quercitrin) & A. eupatoria & [36] & \\
\hline & A. pilosa & [58] & \\
\hline & A. procera & [36] & \\
\hline Quercetin-3-O-Rut (= rutin) & A. eupatoria & [36] & \\
\hline & A. pilosa & [58] & \\
\hline & A. procera & [36] & \\
\hline
\end{tabular}


Table 2. Cont.

\begin{tabular}{|c|c|c|c|}
\hline \multirow{2}{*}{ Compound } & \multirow{2}{*}{ Species } & \multicolumn{2}{|c|}{ Found in [ref.] } \\
\hline & & Herb & Roots \\
\hline Quercetin-3'-O-Glcp & A. pilosa & [37] & \\
\hline \multicolumn{4}{|l|}{ Catechins } \\
\hline \multirow[t]{2}{*}{ Catechin } & A. eupatoria & [34] & \\
\hline & A. pilosa & [58] & \\
\hline Catechin-(5, 6-bc)-4- $\beta$-(4"-hydroxyphenyl)-dihydro-2(H)-pyranone & A. pilosa & [33] & \\
\hline Pilosanol A & A. pilosa & [49] & [59] \\
\hline Pilosanol B & A. pilosa & [49] & [59] \\
\hline Pilosanol C & A. pilosa & [49] & [59] \\
\hline Pilosanol N & A. pilosa & [60] & \\
\hline Isopilosanol A & A. pilosa & [49] & \\
\hline Isopilosanol B & A. pilosa & [49] & \\
\hline Isopilosanol C & A. pilosa & [49] & \\
\hline Pilosandin A & A. pilosa & [49] & \\
\hline Pilosandin B & A. pilosa & [49] & \\
\hline Dehydrodicatechin A & A. pilosa & [33] & \\
\hline Proanthocyanidins & & & \\
\hline Proanthocyanidin B1 & A. eupatoria & [34] & \\
\hline Proanthocyanidin B2 & A. eupatoria & [34] & \\
\hline Proanthocyanidin B3 & A. eupatoria & [34] & \\
\hline Proanthocyanidin B6 & A. eupatoria & [34] & \\
\hline Proanthocyanidin B7 & A. eupatoria & [34] & \\
\hline Proanthocyanidin $\mathrm{C} 1$ & A. eupatoria & [34] & \\
\hline Proanthocyanidin C2 & A. eupatoria & [34] & \\
\hline Flavanonols & & & \\
\hline Dihydrokaempferol & A. pilosa & [47] & \\
\hline Dihydrokaempferol-3-O-Glcp & A. pilosa & [47] & \\
\hline$(2 R, 3 R)$-Dihydrokaempferol-3-O-Glc $p$ & A. pilosa & [61] & \\
\hline (2S,3S)-Dihydrokaempferol-3-O-Glcp & A. pilosa & [61] & \\
\hline$(2 R, 3 S)$-Dihydrokaempferol-3-O-Glcp & A. pilosa & [61] & \\
\hline$(2 S, 3 R)$-Dihydrokaempferol-3-O-Glcp & A. pilosa & [61] & \\
\hline Dihydroquercetin-3-O-Glc $p$ (= glucodistylin) & A. pilosa & [39] & [62] \\
\hline$(2 R, 3 S)$-Dihydroquercetin-3-O-Glc $p$ & A. pilosa & [61] & \\
\hline$(2 R, 3 R)$-Dihydroquercetin-3-O-Glc $p$ & A. pilosa & [61] & \\
\hline$(2 S, 3 R)$-Dihydroquercetin-3-O-Glcp & A. pilosa & [61] & \\
\hline$(2 S, 3 S)$-Dihydroquercetin-3-O-Glcp & A. pilosa & [61] & \\
\hline$(2 R, 3 R)$-Dihydroquercetin-7-O-Glcp & A. pilosa & [61] & \\
\hline$(2 R, 3 R)$-Dihydroquercetin- $4^{\prime}-O-G l c p$ & A. pilosa & [61] & \\
\hline Ellagitannins & & & \\
\hline Agritannin (= 1-O-Gall-2,3-HHDP-Glcp) & A. eupatoria & [35] & \\
\hline & A. pilosa & [37] & \\
\hline Pedunculagin $(=$ 2,3:4,6-Bis-O-HHDP-Glc $p)$ & A. pilosa & [63] & \\
\hline Agrimoniin [= 1-O-\{(2", $3^{\prime}: 4^{\prime}, 6^{\prime}$-bis-O-HHDP-Glc $\left.p\right)$-DHDG $\}-2,3: 4,6-$-bis-O-HHDP-Glc $\left.p\right]$ & A. eupatoria & [45] & \\
\hline & A. pilosa & [63] & [63] \\
\hline & A. procera & [36] & \\
\hline Potentillin (= 1-O-Gall-2,3:4,6-bis-O-HHDP-Glcp) & A. pilosa & [63] & [64] \\
\hline Agrimonic acid A (= 1-O-DHDG-2,3:4,6-bis-O-HHDP-Glcp) & A. pilosa & & [64] \\
\hline Agrimonic acid B (= 1-O-DHDG-2,3:4,6-bis-O-HHDP-Glcp) & A. pilosa & & [64] \\
\hline Sterols & & & \\
\hline$\beta$-Sitosterol & A. pilosa & & [44] \\
\hline Daucosterol & A. pilosa & & [62] \\
\hline Triterpenoids & & & \\
\hline Oleanolic acid & A. pilosa & [65] & \\
\hline $18 \alpha$-Oleanolic acid & A. pilosa & [65] & \\
\hline Ursolic acid & A. asiatica & [32] & \\
\hline & A. pilosa & [66] & \\
\hline $19 \alpha$-Hydroxyursolic acid & A. pilosa & [65] & \\
\hline Pomolic acid & A. pilosa & [66] & \\
\hline $1 \beta$-Hydroxy-2-oxopomolic acid & A. pilosa & [67] & \\
\hline $2 \beta$-Hydroxypomolic acid & A. pilosa & [65] & \\
\hline 3-O-Acetylpomolic acid & A. pilosa & [65] & \\
\hline Tormentic acid & A. pilosa & [66] & [38] \\
\hline Epitormentic acid & A. pilosa & [68] & \\
\hline Corosolic acid & A. pilosa & [66] & \\
\hline
\end{tabular}


Table 2. Cont.

\begin{tabular}{|c|c|c|c|}
\hline \multirow{2}{*}{ Compound } & \multirow{2}{*}{ Species } & \multicolumn{2}{|c|}{ Found in [ref.] } \\
\hline & & Herb & Roots \\
\hline$(1 S, 3 R, 17 R, 18 R, 19 R, 20 R, 22 R)-1,3,19,22-T e t r a h y d r o x y-28-n o r u r s-12-e n-2-o n e$ & A. pilosa & [68] & \\
\hline $1 \beta, 2 \alpha, 3 \beta, 19 \alpha$-Tetrahydroxyurs-12-en-28-oic acid & A. pilosa & & [41] \\
\hline $1 \beta, 2 \beta, 3 \beta, 19 \alpha$-Tetrahydroxyurs-12-en-28-oic acid & A. pilosa & [68] & [41] \\
\hline $1 \beta, 3 \alpha, 19 \alpha$-Trihydroxy-2-oxours-12-en-28-oic acid & A. pilosa & [65] & \\
\hline $1 \beta, 2 \alpha, 19 \alpha$-Trihydroxy-3-oxours-12-en-28-oic acid & A. pilosa & [65] & \\
\hline Maslinic acid & A. pilosa & [65] & \\
\hline Rosamultin $(=2 \alpha, 19 \alpha$-dihydroxyursolic acid-28-O-Glc $p)$ & A. pilosa & [65] & [41] \\
\hline Ziyu-glucoside II (= 19 $\alpha$-hydroxyurs-12-enoic acid-3-O-Arap) & A. pilosa & [65] & \\
\hline \multicolumn{4}{|l|}{ Various groups } \\
\hline Loliolide & A. pilosa & [47] & \\
\hline Tianshic acid & A. pilosa & [33] & \\
\hline Dihydrodehydrodiconifeyl alcohol-9'-O-Glc $p$ & A. pilosa & [37] & \\
\hline
\end{tabular}

Abbreviation used: Ac-acetyl; Araf-arabinofuranose; Arap-arabinopyranose; Bu-butyl;

DHDG—dehydrodigalloyl; Gall—galloyl; Gal $p$ —galactopyranose; Glc $p$ —glucopyranose; GlcA $p$-glucuronopyranose; HHDP-hexahydroxydiphenoyl; Me-methyl; $p$-CouA- $p$-coumaroyl; Rha $p$-rhamnopyranose; Rut-rutinose (6-O-rhamnopyranosyl-glucopyranose); Xylp-xylopyranose.

Flavonols, flavones, and flavanonols were the most studied phenolic compounds of the Agrimonia genus. Some chemical information on A. procera (hydroxycynnamates, flavones, flavonols, and ellagitannins) was provided.

\subsection{Ellagitannins and Other Phenolics of Agrimonia asiatica: LC-MS Seasonal Profile}

To identify potential tendencies in the phenolic constituent profile, Agrimonia asiatica herb samples were collected in May, July, and September, and methanol extracts were obtained. These extracts were analyzed by reversed-phase high-performance liquid chromatography with photodiode array detection and electrospray ionization triple quadrupole mass spectrometric detection (HPLC-PDA-ESI-tQ-MS) in negative ionization mode. HPLC-MS chromatogram is shown in Figure 2 and chromatographic, mass-spectrometric data (ESI-MS), and seasonal presence/content of compounds found in A. asiatica herb are in Table 3.

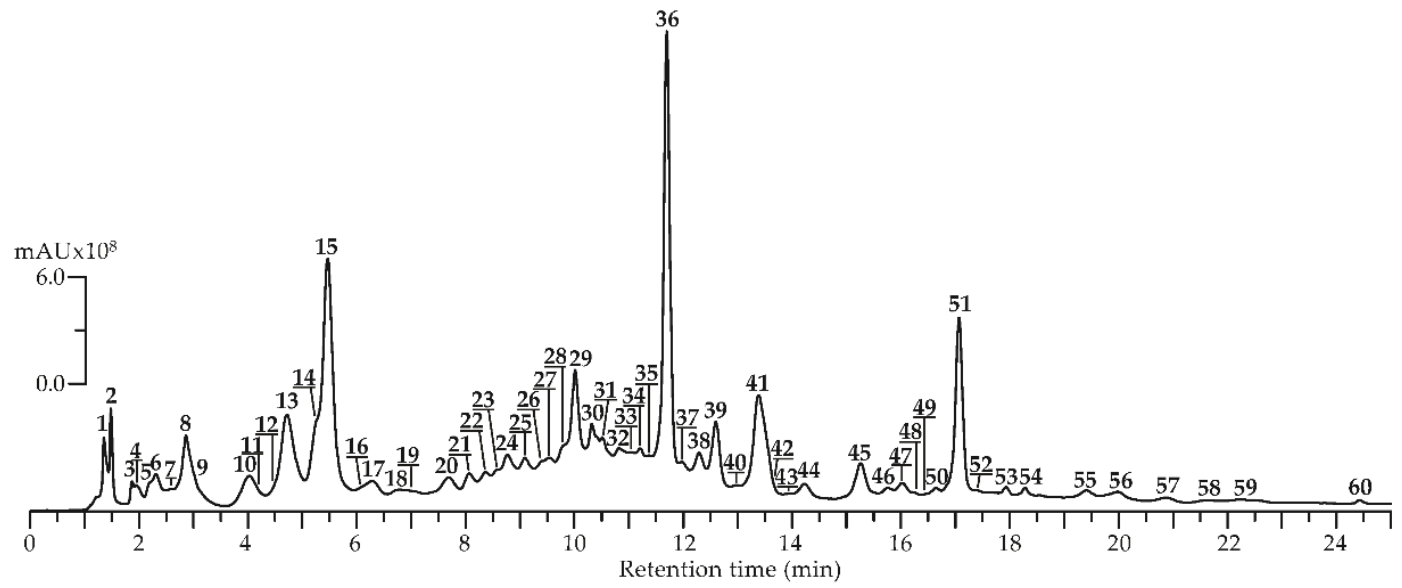

Figure 2. High-Performance Liquid Chromatography with Photodiode Array Detection and Electrospray Ionization Triple Quadrupole Mass Spectrometric Detection (HPLC-PDA-ESI-tQ-MS) chromatogram (Total Ion Chromatogram or TIC mode, negative ionization) of $A$. asiatica herbal extract. Compounds are numbered as listed in Table 3. 
Table 3. Chromatographic $\left(t_{\mathrm{R}}\right)$, mass-spectrometric data (ESI-MS), and seasonal presence/content of compounds 1-60 found in the Agrimonia asiatica herb.

\begin{tabular}{|c|c|c|c|c|c|c|c|}
\hline \multirow{2}{*}{ No } & \multirow{2}{*}{$t_{\mathrm{R}}, \min$} & \multirow{2}{*}{$\begin{array}{l}\text { ESI-MS, } \\
{[\mathrm{M}-\mathrm{H}]^{-}}\end{array}$} & \multirow{2}{*}{$\underset{\mathbf{a}}{\text { Group }}$} & \multirow{2}{*}{ Compound $^{b}$} & \multicolumn{3}{|c|}{ Content, mg/g Dry Plant Weight \pm S.D. } \\
\hline & & & & & May & July & September \\
\hline 1 & 1.38 & 341 & $\mathrm{~S}$ & Hexosyl-O-hexose ${ }^{\mathrm{L}}$ & tr. & tr. & tr. \\
\hline 2 & 1.52 & 331 & GT & 1-O-Galloyl-glucose $\mathrm{s}$ & tr. & n.f. & n.f. \\
\hline 3 & 1.88 & 331 & GT & O-Galloyl-glucose $\mathrm{L}^{\mathrm{L}}$ & $\operatorname{tr}$ & n.f & n.f. \\
\hline 4 & 1.94 & 331 & GT & O-Galloyl-glucose ${ }^{\mathrm{L}}$ & tr. & tr. & n.f. \\
\hline 5 & 2.11 & 331 & GT & O-Galloyl-glucose $\mathrm{L}$ & tr. & n.f. & n.f. \\
\hline 6 & 2.36 & 183 & $\mathrm{~V}$ & 2-Pyrone-4,6-dicarboxylic acid S & $1.42 \pm 0.03$ & $2.05 \pm 0.04$ & $2.11 \pm 0.03$ \\
\hline 7 & 2.67 & 783 & ET & Pedunculagin S & tr. & tr. & tr. \\
\hline 8 & 2.91 & 353 & $\mathrm{HC}$ & 4-O-Caffeoylquinic acid $\mathrm{S}$ & $15.63 \pm 0.32$ & $18.59 \pm 0.37$ & $16.37 \pm 0.31$ \\
\hline 9 & 3.22 & 783 & ET & Casuariin $\mathrm{s}$ & tr. & tr. & tr. \\
\hline 10 & 4.01 & 577 & $\mathrm{P}$ & Procyanidin $\mathrm{B}_{1} \mathrm{~S}$ & tr. & $\operatorname{tr}$. & tr. \\
\hline 11 & 4.26 & 785 & ET & Tellimagrandin $\mathrm{I}_{1} \mathrm{~S}$ & tr. & tr. & n.f. \\
\hline 12 & 4.51 & 577 & $\mathrm{P}$ & Procyanidin B3 ${ }^{S}$ & tr. & tr. & tr. \\
\hline 13 & 4.76 & 289 & $\mathrm{C}$ & Catechin $\mathrm{s}$ & $20.19 \pm 0.41$ & $22.27 \pm 0.45$ & $11.63 \pm 0.19$ \\
\hline 14 & 5.31 & 353 & $\mathrm{HC}$ & 5-O-Caffeoylquinic acid $\mathrm{S}$ & tr. & tr. & tr. \\
\hline 15 & 5.51 & 305 & $\mathrm{C}$ & Epigallocatechin $\mathrm{S}$ & $27.02 \pm 0.54$ & $65.15 \pm 1.30$ & $36.11 \pm 0.72$ \\
\hline 16 & 6.09 & 577 & $\mathrm{P}$ & Procyanidin B2 $\mathrm{s}$ & tr. & tr. & tr. \\
\hline 17 & 6.33 & 447 & $\mathrm{~V}$ & Ellagic acid-O-methyl ester-O-pentoside ${ }^{\mathrm{L}}$ & n.f. & tr. & tr. \\
\hline 18 & 6.59 & 937 & ET & Tri-O-galloyl-O-hexahydroxydiphenoyl-glucose ${ }^{\mathrm{L}}$ & tr. & tr. & n.f. \\
\hline 19 & 6.92 & 785 & ET & Tellimagrandin $\mathrm{I}_{2} \mathrm{~S}$ & tr. & tr. & n.f. \\
\hline 20 & 7.54 & 577 & $\mathrm{P}$ & Procyanidin B (dimer) ${ }^{\mathrm{L}}$ & $\operatorname{tr}$. & tr. & tr. \\
\hline 21 & 8.03 & 783 & ET & Bis-O-hexahydroxydiphenoyl-glucose $\mathrm{L}^{\mathrm{L}}$ & $\operatorname{tr}$ & $\operatorname{tr}$ & n.f. \\
\hline 22 & 8.20 & 785 & ET & Di-O-galloyl-O-hexahydroxydiphenoyl-glucose $\mathrm{L}^{\mathrm{L}}$ & $\operatorname{tr}$ & tr. & n.f. \\
\hline 23 & 8.49 & 289 & $\mathrm{C}$ & Epicatechin $\mathrm{s}$ & tr. & tr. & tr. \\
\hline 24 & 8.71 & 337 & ET & Hexahydroxydiphenic acid ${ }^{\mathrm{L}}$ & tr. & tr. & n.f. \\
\hline 25 & 9.08 & 785 & ET & Di-O-galloyl-O-hexahydroxydiphenoyl-glucose ${ }^{\mathrm{L}}$ & $\operatorname{tr}$. & $\operatorname{tr}$. & n.f. \\
\hline 26 & 9.46 & 515 & $\mathrm{HC}$ & 1,3-Di-O-caffeoyquinic acid ${ }^{S}$ & tr. & tr. & tr. \\
\hline 27 & 9.54 & 783 & ET & Bis-O-hexahydroxydiphenoyl-glucose ${ }^{\mathrm{L}}$ & tr. & tr. & n.f. \\
\hline 28 & 9.69 & 463 & $\mathrm{~F}$ & 6-Hydroxyluteolin-7-O-Glc $\mathrm{s}$ & n.f. & tr. & n.f. \\
\hline 29 & 10.02 & 783 & ET & Bis-O-hexahydroxydiphenoyl-glucose ${ }^{\mathrm{L}}$ & tr. & $\operatorname{tr}$. & n.f. \\
\hline 30 & 10.27 & 577 & $\mathrm{P}$ & Procyanidin B (dimer) ${ }^{\mathrm{L}}$ & n.f. & $\operatorname{tr}$. & tr. \\
\hline 31 & 10.42 & 937 & ET & Tellimagrandin $\mathrm{II}_{1} \mathrm{~s}$ & tr. & tr. & n.f. \\
\hline 32 & 10.71 & 935 & ET & Potentillin $\mathrm{S}$ & tr. & tr. & tr. \\
\hline 33 & 11.01 & 1103 & ET & Agrimonic acid A $\mathrm{S}$ & tr. & tr. & n.f. \\
\hline 34 & 11.12 & 1871 & ET & Gemin As & tr. & tr. & tr. \\
\hline 35 & 11.48 & 1103 & ET & Agrimonic acid B $\mathrm{S}$ & tr. & tr. & n.f. \\
\hline 36 & 11.63 & 1869 & ET & Agrimoniin $\mathrm{S}$ & $82.59 \pm 1.67$ & $114.18 \pm 2.37$ & $27.32 \pm 0.54$ \\
\hline 37 & 11.97 & 441 & $\mathrm{C}$ & Epicatechin gallate $\mathrm{S}$ & tr. & tr. & tr. \\
\hline 38 & 12.27 & 301 & $\mathrm{~V}$ & Ellagic acid $\mathrm{S}$ & $3.60 \pm 0.06$ & $5.31 \pm 0.12$ & $34.62 \pm 0.69$ \\
\hline 39 & 12.51 & 609 & $\mathrm{~F}$ & Quercetin-3-O-(6"-O-rhamnosyl)-glucoside $\mathrm{S}$ & $8.06 \pm 0.17$ & $16.32 \pm 0.31$ & $17.83 \pm 0.35$ \\
\hline 40 & 12.98 & 937 & ET & Tellimagrandin $\mathrm{II}_{2} \mathrm{~S}$ & tr. & tr. & n.f. \\
\hline 41 & 13.41 & 463 & $\mathrm{~F}$ & Quercetin-3-O-glucoside $^{\mathrm{S}}$ & $9.53 \pm 0.17$ & $29.80 \pm 0.61$ & $24.18 \pm 0.48$ \\
\hline 42 & 13.50 & 477 & $\mathrm{~F}$ & Quercetin-3-O-glucuronide $\mathrm{S}$ & tr. & tr. & tr. \\
\hline 43 & 13.89 & 447 & $\mathrm{~F}$ & Luteolin-7-O-glucoside $\mathrm{S}$ & tr. & tr. & tr. \\
\hline 44 & 14.21 & 461 & $\mathrm{~F}$ & Luteolin-7-O-glucuronide $\mathrm{S}$ & $2.16 \pm 0.04$ & $4.21 \pm 0.09$ & $4.69 \pm 0.08$ \\
\hline 45 & 15.20 & 549 & $\mathrm{~F}$ & Quercetin-3-O-(6"-O-malonyl)-glucoside $\mathrm{S}$ & $6.37 \pm 0.12$ & $4.14 \pm 0.08$ & $0.92 \pm 0.02$ \\
\hline 46 & 15.67 & 515 & $\mathrm{HC}$ & 3,5-Di-O-caffeoyquinic acid $\mathrm{S}$ & $2.40 \pm 0.04$ & $2.59 \pm 0.05$ & $0.31 \pm 0.01$ \\
\hline 47 & 16.01 & 593 & $\mathrm{~F}$ & Kaempferol-3-O-(6"-O-rhamnosyl)-glucoside $\mathrm{S}$ & $7.53 \pm 0.15$ & $9.27 \pm 0.19$ & $11.36 \pm 0.21$ \\
\hline 48 & 16.20 & 447 & $\mathrm{~F}$ & Quercetin-3-O-rhamnoside $\mathrm{S}$ & tr. & tr. & tr. \\
\hline 49 & 16.51 & 447 & $\mathrm{~F}$ & Kaempferol-3-O-glucoside ${ }^{S}$ & $\operatorname{tr}$ & tr. & tr. \\
\hline 50 & 16.55 & 431 & $\mathrm{~F}$ & Apigenin-7-O-glucoside $\mathrm{S}$ & tr. & $0.75 \pm 0.02$ & $0.82 \pm 0.02$ \\
\hline 51 & 17.08 & 445 & $\mathrm{~F}$ & Apigenin-7-O-glucuronide $\mathrm{S}$ & $22.18 \pm 0.44$ & $47.22 \pm 0.94$ & $56.14 \pm 1.14$ \\
\hline 52 & 17.27 & 533 & $\mathrm{~F}$ & Kaempferol-O-malonyl-O-hexoside $\mathrm{L}$ & tr. & tr. & tr. \\
\hline 53 & 17.91 & 533 & $\mathrm{~F}$ & Luteolin-3-O-(6"-O-malonyl)-glucoside $\mathrm{S}$ & $2.10 \pm 0.04$ & $0.85 \pm 0.02$ & n.f. \\
\hline 54 & 18.23 & 431 & $\mathrm{~F}$ & Kaempferol-3-O-rhamnoside $\mathrm{S}$ & $0.21 \pm 0.00$ & $0.59 \pm 0.01$ & $0.73 \pm 0.01$ \\
\hline 55 & 19.43 & 609 & $\mathrm{~F}$ & Quercetin-3-O-(6"-O-p-coumaroyl)-glucoside $\mathrm{S}$ & tr. & tr. & tr. \\
\hline 56 & 20.01 & 593 & $\mathrm{~F}$ & Kaempferol-3-O-(6"-O-p-coumaroyl)-glucoside $\mathrm{S}$ & $\operatorname{tr}$. & tr. & $\operatorname{tr}$ \\
\hline 57 & 20.87 & 329 & $\mathrm{~V}$ & Ellagic acid di-O-methyl ester ${ }^{\mathrm{L}}$ & $\operatorname{tr}$ & $\operatorname{tr}$ & tr. \\
\hline 58 & 21.63 & 593 & $\mathrm{~F}$ & Luteolin-7-O-(6"-O-p-coumaroyl)-glucoside $\mathrm{S}$ & tr. & tr. & n.f. \\
\hline 59 & 22.24 & 517 & $\mathrm{~F}$ & Apigenin-7-O-(6"-O-p-malonyl)-glucoside $\mathrm{S}$ & tr. & tr. & tr. \\
\hline 60 & 24.43 & 577 & $\mathrm{~F}$ & Apigenin-7-O-(6"-O-p-coumaroyl)-glucoside $\mathrm{S}$ & tr. & $\operatorname{tr}$ & tr. \\
\hline
\end{tabular}

${ }^{a}$ Chemical group of compound: C-catechins, ET-ellagitannins, F-flavones/flavonols, GT—gallotannins, HC-hydroxycinnamates, $\mathrm{P}$ - procyanidins, $\mathrm{S}$ - sugars, V-various compounds. ${ }^{\mathrm{b}}$ The identification of compounds was realized on comparison of retention time, UV and MS spectral data with a reference standard $\left({ }^{\varsigma}\right)$ or interpretation of UV and MS spectral data and comparison with literature data $\left({ }^{\mathrm{L}}\right)$. tr.- trace, n.f.-not found.

By comparison the retention times $\left(t_{R}\right)$, ultraviolet (UV) and ESI-MS spectra with those of reference substances and literature data, 60 compounds were discovered in all plant samples, including four catechins $(\mathbf{1 3}, \mathbf{1 5}, \mathbf{2 3}, \mathbf{3 7}), 18$ ellagitannins $(\mathbf{7 , 9 , 1 1 , 1 8 , 1 9 , 2 1 , 2 2 , 2 4 , 2 5 , 2 7 , 2 9 , 3 1 - 3 6 , 4 0 ) , ~} 20$ 


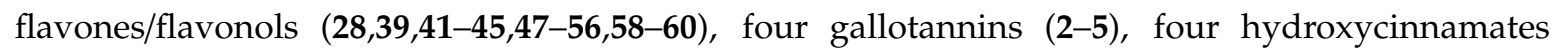
$(8, \mathbf{1 4}, \mathbf{2 6}, \mathbf{4 6})$, five procyanidins $(\mathbf{1 0}, \mathbf{1 2}, \mathbf{1 6}, 20,30)$, one sugar $(1)$ and four various phenolics $(6,17,38,57)$.

Four chromatographic peaks with a similar UV profile typical of caffeoyl derivatives (caffeoylquinic acids) were detected in A. asiatica. The charge on deprotonated ions made it possible to identify them as mono-O-caffeoylquinic acids with $\mathrm{m} / \mathrm{z} 353(\mathbf{8 , 1 4})$ and di-O-caffeoylquinic acids with $\mathrm{m} / \mathrm{z}$ 515 (26,46). Compared with reference standards 4-O-caffeoylquinic (8), 5-O-caffeoylquinic (14), 1,3-di-O-caffeoylquinic (26), 3,5-di-O-caffeoylquinic acids (46) were found. Mono-O-caffeoylquinic acids $(\mathbf{8}, \mathbf{1 4})$ were detected in $A$. eupatoria and A. procera previously $[36,45]$, while di-O-caffeoylquinic acids $(\mathbf{2 6}, \mathbf{4 6})$ were discovered in the genus Agrimonia for the first time. It should be noted that all hydroxycinnamates were present in all seasonal samples of $A$. asiatica. The content of 4 -O-caffeoylquinic acid (8) reached a maximum in the July sample $(18.59 \mathrm{mg} / \mathrm{g})$ and slightly decreased in the September sample $(16.37 \mathrm{mg} / \mathrm{g})$. However, the content of 3,5-di-O-caffeoylquinic acid (46) significantly decreased more than 8 times in September samples $(0.31 \mathrm{mg} / \mathrm{g})$ compared with July samples $(2.59 \mathrm{mg} / \mathrm{g})$.

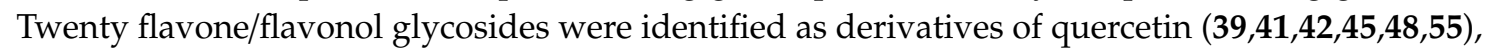
kaempferol $(47,49,52,54,56)$, apigenin $(50,51,59,60)$ and luteolin $(28,43,44,53,58)$. Flavonol glycosides were represented as derivatives of quercetin and kaempferol $[36,56]$.

Quercetin-3-O-(6"-O-rhamnosyl)-glucoside (39), quercetin-3-O-glucoside (isoquercitrin, 41), quercetin-3-O-glucuronide (miquelianin, 42), quercetin-3-O-(6"-O-malonyl)-glucoside (45), quercetin-3-O-rhamnoside (quercitrin, 48), quercetin-3-O-(6"-O-p-coumaroyl)-glucoside (55) standards made it possible to unambiguously identify these six flavonol glycosides in A. asiatica extract. Isoquercitrin (41) and quercitrin (48) have already been characterized as components of A. eupatoria, A. procera and A. pilosa [36,58]. Whereas quercetin-3-O-(6"-O-rhamnosyl)-glucoside (39), quercetin-3-O-glucuronide (miquelianin, 42), quercetin-3-O-(6"-O-malonyl)-glucoside (45), quercetin-3-O-(6"-O-p-coumaroyl)-glucoside (55) were discovered in genus Agrimonia for the first time. Four kaempferol derivatives were detected as kaempferol-3-O-(6"-O-rhamnosyl)-glucoside (47), kaempferol-3-O-glucoside (astragalin, 49), kaempferol-3-O-rhamnoside (afzelin, 54), kaempferol-3-O-(6"-O-p-coumaroyl)-glucoside (tiliroside, 56) by comparison with standards. Compound $\mathbf{5 2}$ was identified as kaempferol-O-malonyl-O-hexoside in accordance with UV and ESI-MS spectra and literature data. The negative mass spectrum displayed a deprotonated ion $[\mathrm{M}-\mathrm{H}]^{-}$with $\mathrm{m} / \mathrm{z}$ 533, as well as demalonated fragment with $m / z 447$, and the aglycone fragment with $m / z 285$ caused the removal of a hexose particle [69]. Astragalin (49) was revealed earlier in A. eupatoria [55], A. pilosa [56], A. procera [36]. Afzelin (54) was detected in A. eupatoria [55] and A. pilosa [56]. Tiliroside (56) was previously identified in A. eupatoria [34] and A. pilosa [57]. Kaempferol-3-O-(6"-O-rhamnosyl)-glucoside (47) was discovered in $A$. asiatica for the first time in this work. Five luteolin derivatives were characterized as 6-hydroxyluteolin-7-O-glucoside (28), luteolin-7-O-glucoside (cynaroside, 43), luteolin-7-O-glucuronide (44), luteolin-3-O-(6"-O-malonyl)-glucoside (53), luteolin-7-O-(6"-O-p-coumaroyl)-glucoside (58) after comparison of $t_{R}, U V$, and mass-spectrometric data (MS, MS/MS) with the reference standards. Four apigenin derivatives: apigenin-7-O-glucoside (cosmosiin, 50), apigenin-7-O-glucuronide (51), apigenin-7-O-(6"-O-p-malonyl)-glucoside (59) and apigenin-7-O-(6"-O-p-coumaroyl)-glucoside (60) were detected after comparison with standards. Two luteolin derivatives cynaroside (43) and luteolin-7-O-glucuronide (44) and two apigenin derivatives cosmosiin (50) and apigenin-7-O-glucuronide (51) have already been characterized as components of A. eupatoria, A. pilosa and A. procera [36,45,51-53]. 6-hydroxyluteolin-7-O-glucoside (28), luteolin-3-O-(6"-O-malonyl)-glucoside (53), luteolin-7-O-(6"-O-p-coumaroyl)-glucoside (58), apigenin-7-O-(6"-O-p-malonyl)-glucoside (59) and apigenin-7-O-(6"-O-p-coumaroyl)-glucoside (60) were discovered in the Agrimonia genus for the first time. According to the data obtained, the presence of flavone/flavonol glycosides is observed in all seasonal samples of $A$. asiatica. However, there were curious differences between the different derivatives of flavones and flavonols. It was noticed that the presence of some flavones, in particular apigenin derivatives, was detected in all seasonal samples of the A. asiatica and even an increase in the content of some compounds (apigenin-7-O-glucoside, 
50; apigenin-7-O-glucuronide, 51) was observed in the September samples. Other representatives of flavones, luteolin derivatives, were not present in all seasonal samples of the A. asiatica. It is difficult to trace the accumulation trend for quercetin derivatives, since the peak accumulation of some compounds occurs in May samples (quercetin-3-O-(6"-O-malonyl)-glucoside, 45), July samples (quercetin-3-O-glucoside, 41) and even September samples (quercetin-3-O-(6"-O-rhamnosyl)-glucoside, 39). Other representatives of flavonols, kaempferol derivatives were present in all seasonal samples, and the maximum content of some compounds is observed in September samples (kaempferol-3-O-(6"-O-rhamnosyl)-glucoside, 47; kaempferol-3-O-rhamnoside, 54).

Hydrolyzable tannins of $A$. asiatica were presented by both groups-ellagitannins and gallotannins. Ellagitannins are esters of hexahydroxydiphenoyl (HHDP) groups with a sugar core and often contain galloyl groups. Gallotannins consist of a sugar substituted only with galloyl groups [70]. Ellagitannins of various types: C-glucosidic (casuariin, 9), hexahydroxydiphenoyl glucose (pedunculagin, 7), hexahydroxydiphenoyl-galloyl-glucose (tellimagrandins $\mathrm{I}_{1}(\mathbf{1 1}), \mathrm{I}_{2}(\mathbf{1 9}), \mathrm{II}_{1}(31)$, $\mathrm{II}_{2}(40)$, potentillin (32)), dehydrodigalloyl (agrimoniin (36), gemin A (34), agrimonic acids A (33) and B (35)) were found in $A$. asiatica herb by comparison their properties with standards. Compound 18 was identified as tri-O-galloyl-O-hexahydroxydiphenoyl glucose in accordance with UV, mass-spectrometric data (MS, MS/MS), and literature data. The negative mass spectrum showed a deprotonated ion $[\mathrm{M}-\mathrm{H}]^{-}$ with $\mathrm{m} / \mathrm{z} 937$ and the fragmentation patterns corresponded to the loss of gallic acid units, HHDP units, and the loss of a glucosyl moiety [71]. Di-O-galloyl-O-hexahydroxydiphenoyl-glucose $(\mathbf{2 2}, \mathbf{2 5})$ was identified based on the relationship between the supposed parent deprotonated ion at $m / z 785$ and fragments having $\mathrm{m} / \mathrm{z}$ values corresponding to the losses of galloyl units, glucosyl units, and HHDP units [72]. Compounds 21, 27, 29 were identified as bis-O-hexahydroxydiphenoyl glucose and were possible isomers of pedunculagin (7) or casuariin (9). These compounds were isomeric and displayed a parent peak at $m / z 783$, yielding main fragment ions at $m / z 481$ [(M-H)-302] $]^{-}$loss of HHDP) and 301 [(M-H)-482 $]^{-}$(loss of HHDP-glucose), whose fragmentation pattern corresponds to a bis-HHDP-glucose structure [73,74]. Compound 24 was identified as hexahydroxydiphenic acid in accordance with UV and ESI-MS spectra and literature data. The negative mass spectrum showed a deprotonated ion [M-H] $]^{-}$with $\mathrm{m} / \mathrm{z} 337$ [71]. Pedunculagin (7), agrimoniin (36), potentillin (32), agrimonic acids A (33), and B (35) were found in A. pilosa earlier [63,64]. Agrimoniin (36) was also found in A. eupatoria [45] and A. procera [36]. Thus casuariin (9), tellimagrandins $\mathrm{I}_{1}$ (11), $\mathrm{I}_{2}(\mathbf{1 9}), \mathrm{I}_{1}$ (31), $\mathrm{II}_{2}$ (40), and gemin A (34) were described for the genus Agrimonia for the first time.

Significant changes in the ellagitannin seasonal profile were observed. It was noticed that the presence of some ellagitannins was detected in all seasonal samples of A. asiatica, while other ellagitannins were revealed only in samples of a certain season. Thus, the presence of ellagitannin of hexahydroxydiphenoyl glucose pedunculagin (7) and C-glucosidic casuariin (9) groups was noticed for all seasonal samples of A. asiatica (May, July, September), while ellagitannins of hexahydroxydiphenoyl-galloyl-glucose group tellimagrandins $\mathrm{I}_{1}(\mathbf{1 1}), \mathrm{I}_{2}(\mathbf{1 9}), \mathrm{II}_{1}(\mathbf{3 1}), \mathrm{II}_{2}(\mathbf{4 0})$ were revealed only in May and July samples of $A$. asiatica. It may be because $C$-glucosidic ellagitannin casuariin (9) is supposed to be biosynthesized from pedunculagin (7) due to its almost unchanged combination with pedunculagin and/or tellimagrandin $\mathrm{I}_{1}(\mathbf{1 1}), \mathrm{I}_{2}(\mathbf{1 9})$, and is a plausible precursor. Pedunculagin (7) can be considered as the product of oxidative binding between two galloyl groups in tellimagrandin I $(\mathbf{1 1}, \mathbf{1 9})$. A similar trend was observed for the seasonal fluctuations in the content of these compounds in leaves of Liquidambar formosana (Hamamelidaceae) [75]. The synthesis of casuariin (9) in the leaves of L. formosana in autumn was followed by a decrease and then by the complete disappearance of tellimagrandins I $(\mathbf{1 1}, \mathbf{1 9})$ and II $(\mathbf{3 1}, \mathbf{4 0})$, which were abundant in the young leaf in spring. This seasonal change is in accordance with the probability theory that this $C$-glucosidic tannin is biosynthesized from the gallotannins and ellagitannins which were found in the young leaves [76].

Ellagitannin agrimoniin (36) was the dominant compound of A. asiatica herb. Its content was the highest in July samples $(114.18 \mathrm{mg} / \mathrm{g})$ during mass flowering and then sharply decreased in September samples by more than 4 times $(27.32 \mathrm{mg} / \mathrm{g})$. A similar phenomenon was observed for the 
genus Alchemilla. In A. vulgaris and A. mollis the content of agrimoniin (36) increased by the end of the mass flowering period by more than $100 \%$ [77]. The presence of potentillin (32) in all seasonal samples is probably because it is a main intermediate metabolite in the production of agrimoniin. Agrimoniin (36) is a dimeric ellagitannin in which hydroxyl groups on the two glucose cores are esterified by a dehydrodigalloyl group and four hexahydroxydiphenoyl groups. Agrimoniin (36) may have been formed by intermolecular $\mathrm{C}-\mathrm{O}$ oxidative binding between galloyl groups in two molecules of potentillin (32) [64].

Gallotannins were the second group of hydrolysable tannins and were presented in A. asiatica herb by 1-O-galloyl glucose $(2)$ and $O$-galloyl-glucoses $(3,4,5)$. Gallotannins are turned into to ellagitannins through oxidative binding of the galloyl groups. Several ellagitannin derivatives form dimers after intermolecular binding with other hydrolyzable tannin molecules [78]. The content of galloylglucoses $(2,3,5)$ was higher in May samples than in the samples collected in July during the mass flowering period. Galloylglucoses are predecessors to other more complex hydrolyzable tannins. Consimilar season tendency for galloylglucoses has been reported in the investigations of Geranium sylvaticum [79], Quercus robur [80], and Betula pubescens [81]. In accordance with literature data, the structures of tree leaf hydrolyzable tannins vary within the vegetation period. These variations follow the biogenetic pathway. Thus, the simple galloylglucose predecessors revealed in springtime are converted to more complex compounds. These complex structures contain more galloyl and oxidatively binded HHDP groups during the vegetation season. In contrast to woody plants, there is an assumption that hydrolyzable tannins of herbaceous plants generally preserve their chemical structures until leaves destruction [82]. Nevertheless, research results demonstrate that hydrolyzable ellagitannins can be converted during the vegetation season in herbaceous plants, as has been observed in the case herb of $A$. asiatica. The contents of galloylglucoses, that are predecessors to more complex hydrolyzable tannins, were higher in May samples, and the amount of further modified ellagitannins increased towards the flowering period (July sample). So, to maximize the isolated content of ellagitannins, A. asiatica herb must be harvested during the flowering period (July).

\section{3. $\alpha$-Glucosidase Inhibiting Activity of A. asiatica Extract: HPLC Activity-Based Profiling}

In natural product research, the finding of new bioactive components useful for drug discovery is the main goal. This requires the early detection of bioactive substances in complex matrices to isolate only the necessary components [83]. A common procedure involves searching for substances from complex matrices such as extracts, including biological screening followed by activity guided fractionation [84]. Such approaches mainly consist of the post-column collecting of microfractions, drying them for solvent removal, and biological assessment of the microfractions. Ultimately, the activity measured for each microfraction, is compared to the HPLC profile to reveal the LC peaks that are responsible for the activity [83].

To characterize the chemical profile of $A$. asiatica herb extract microcolumn reversed-phase HPLC-PDA procedure was applied (Figure 3a). We compared the data received with those of reference substances 4-O-caffeoylquinic acid (8), epigallocatechin (15), agrimoniin (36), ellagic acid (38), quercetin-3-O-(6"-O-rhamnosyl)-glucoside (39), and quercetin-3-O-glucoside (isoquercitrin, 41) were revealed. For identification, the components of interest of $A$. asiatica herb with high $\alpha$-glucosidase inhibitory activity, the extract was subjected by HPLC activity-based profiling. To detect of anti- $\alpha$-glucosidase inhibitors in A. asiatica herb extract, the procedure of small-scale semi-preparative microfractionation by reversed-phase HPLC was applied. This resulted in 48 fractions of $30 \mathrm{~s}$ each that were transferred to a deep-well microtiter plate. Subsequently, microfractions were dried and redissolved in a buffer solution. Thereafter, an enzyme solution was added to estimate the inhibition of $\alpha$-glucosidase by the eluate. The optical densities at $400 \mathrm{~nm}\left(\mathrm{~A}_{400}\right)$ as $\left(1-\mathrm{A}_{400}\right)$ values of microfractions after post-column derivatization are displayed in Figure $3 \mathrm{~b}$. 

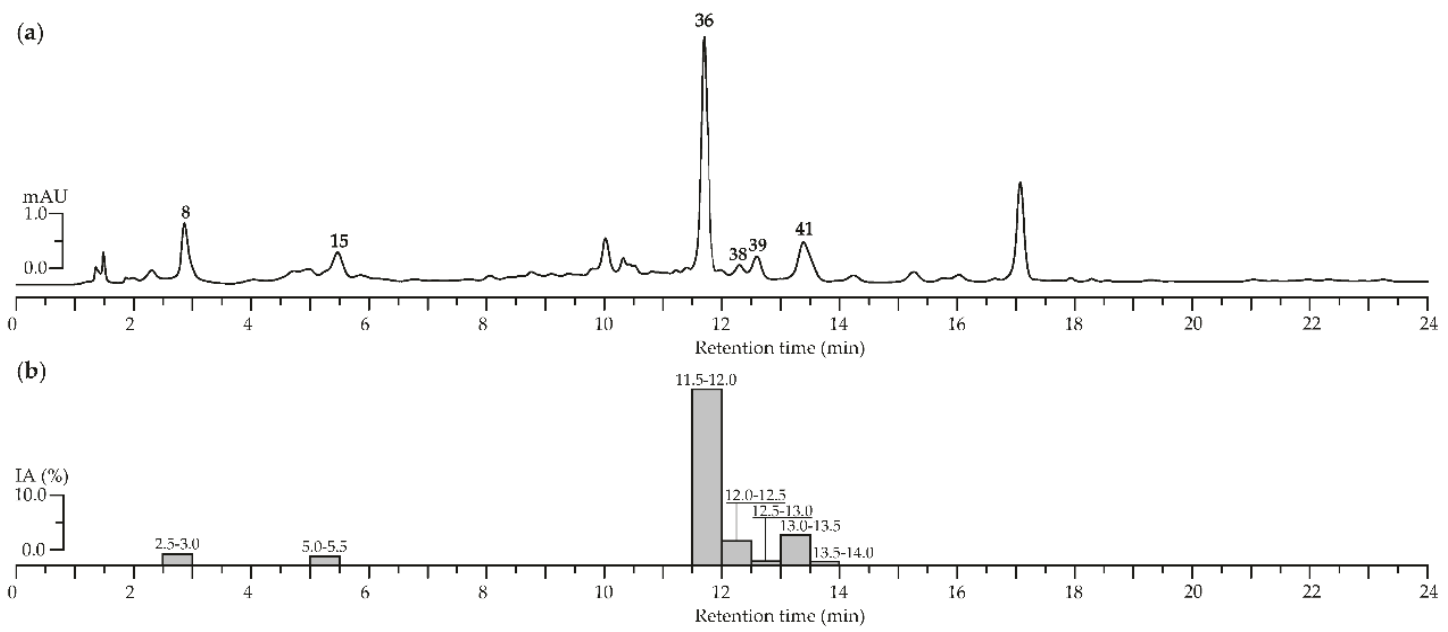

Figure 3. (a) High-Performance Liquid Chromatography with Photodiode Array Detection (HPLC-PDA) chromatograms of $A$. asiatica extract at $270 \mathrm{~nm}$. Compounds numbered as $\mathbf{8}$ (4-O-caffeoylquinic acid), 15 (epigallocatechin), 36 (agrimoniin), 38 (ellagic acid), 39 (quercetin-3-O-(6"-O-rhamnosyl)-glucoside), and 41 (quercetin-3-O-glucoside). (b) $\alpha$-Glucosidase inhibiting activity (IA, as a percentage) of HPLC fractions of $A$. asiatica extract after microfractionation technique. Bars are labeled as retention time range ( $\mathrm{min})$.

The main inhibition was observed in the time window of 11.5 to $13.5 \mathrm{~min}$, corresponding to 5 fractions. The $\alpha$-glucosidase inhibition activity measured for each microfraction was compared with the HPLC profile to localize the corresponding chromatogram peaks. The most pronounced inhibition of $\alpha$-glucosidase was observed for agrimoniin (36), while less significant inhibition was revealed for ellagic acid (38) and quercetin-3-O-glucoside (isoquercitrin, 41). In this way, ellagitannin agrimoniin (36) from the extract of $A$. asiatica herb is considered the responsible compound for the inhibition of $\alpha$-glucosidase previously detected in screening investigations. Earlier we proved high inhibitory activity of agrimoniin (36) from Comarum palustre herb extract relative to $\alpha$-glucosidase [29]. A possible mechanism of the inhibitory activity against $\alpha$-glucosidase of ellagitannins from the extract analyzed could be tannin-protein coupling. There are changes in the conformation of the enzyme and therefore the activity of $\alpha$-glucosidase decreases due to association and precipitation $[85,86]$.

Thus, the microfractionation of crude plant extracts, particularly A. asiatica, makes it possible to directly evaluate the bioactivities of LC-peaks within their biological matrices. By miniaturizing enzymatic assays in a deep-well microtiter plate, HPLC-based biological activity profiling has become accessible for many types of analyzes and is supplementary to metabolite profiling, enabling bioactive components to easily be localized and partly identified. In this way, such methods should accelerate the detection of compounds from plant extracts and help rationalize the classical approach based on biological activity, which was considered as too slow and difficult for the pharmaceutical industries [87].

\subsection{Stability of A. asiatica Phenolics in Water Media: Comparison of Infusion and Decoction Composition and} Their Anti- $\alpha$-Glucosidase Activity

When natural biologically active components are extracted from plant materials, the main disadvantages are associated with possible decomposition and/or changes in chemical structures. As such, the extraction procedures must be chosen carefully. So, we decided to compare phenolic content in A. asiatica herb infusions and decoctions depending on the method of obtaining herbal tea (Table 4). Herbal drinks are beverages made from the infusion or decoction of herbs, spices, fruits, or other plant materials, that are served cold or hot [88]. In decoction, the plant material is boiled, or kept at high temperatures, in water for a certain time. An infusion is obtained by adding water to a certain amount of plant material and leaving the mix to brew, similar to the preparation of tea [89]. 
Table 4. Phenolic content in agrimonia tea infusions and decoctions (mg/100 $\mathrm{mL} \pm$ S.D.) and their $\alpha$-glucosidase inhibiting potential $\left(\alpha \mathrm{GIP}, \mathrm{IC}_{50}, \mu \mathrm{g} / \mathrm{mL}\right)$.

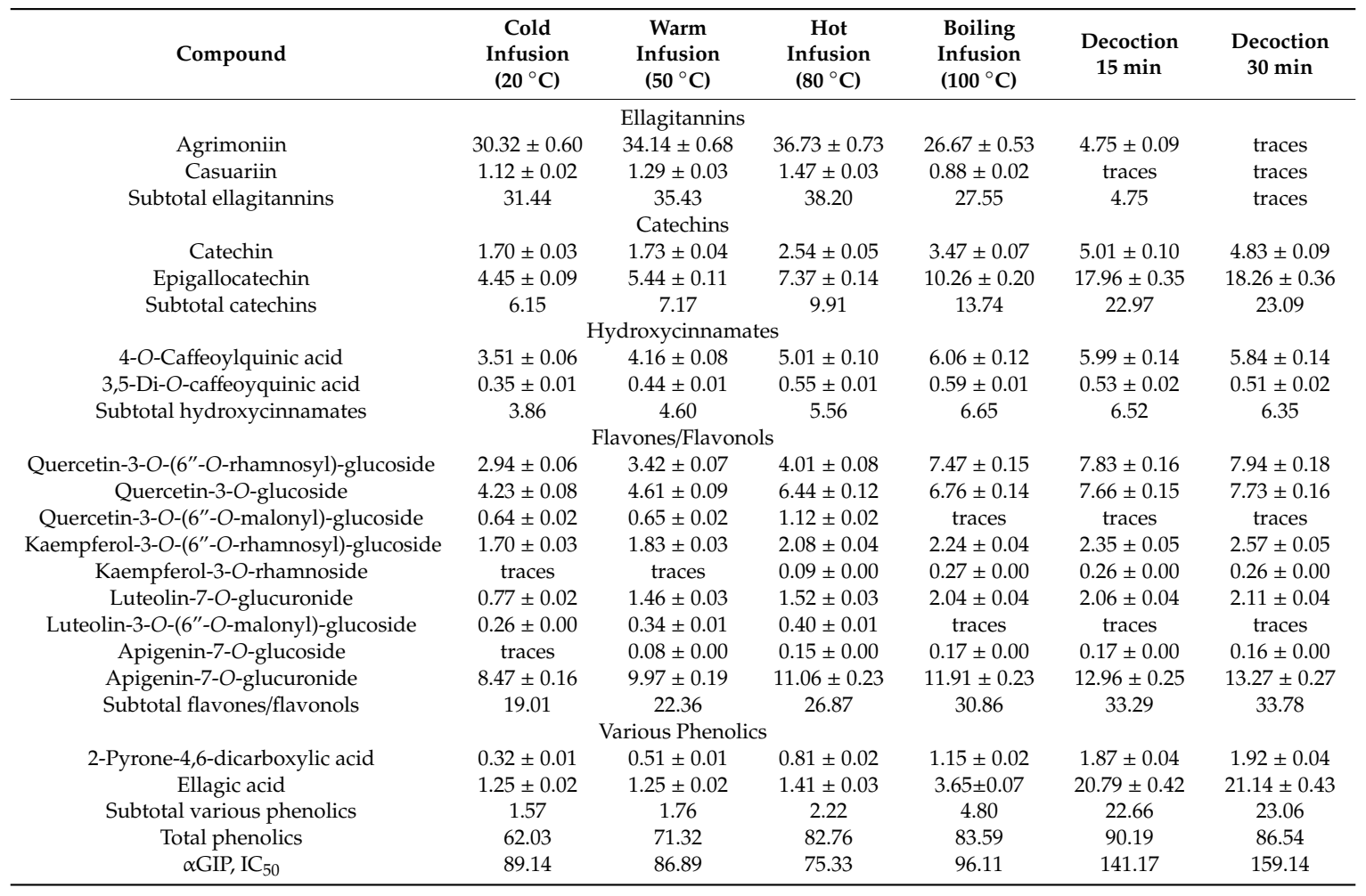

The maximal content of ellagitannins $(38.20 \mathrm{mg} / 100 \mathrm{~mL})$ was observed in the infusion obtained by the extraction of herb A. asiatica with water at $80^{\circ} \mathrm{C}$. On the contrary, in the decoction obtained by extraction of herb A. asiatica for $15 \mathrm{~min}$, a significant decrease in ellagitannins $(4.75 \mathrm{mg} / 100 \mathrm{~mL})$ content was revealed. During extraction for $30 \mathrm{~min}$, complete degradation and trace amounts of ellagitannins were detected. However, for both decoctions, a significant increase in ellagic acid was observed. It has previously been reported earlier that ellagitannins may be unstable when processed at higher temperatures, leading to their degradation to ellagic acid [90]. Our findings confirm early data for A. eupatoria herb that the degradation products of agrimoniin are not present in large quantities if the effect of temperature is limited in time $[36,45]$.

The total content of hydroxycinnamates gradually increased in infusions from $3.86 \mathrm{mg} / 100 \mathrm{~mL}$ to $6.65 \mathrm{mg} / 100 \mathrm{~mL}$ as the temperature of the extract increased. Then, when analyzing the decoctions, a slight decrease in the content of hydroxycinnamates was revealed. Li et al. had previously studied the thermal stability of mono- and di-caffeoylquinic acids [91]. It was found that after heating for $1 \mathrm{~h}$, the amount of three mono-caffeoylquinic acids was reduced by about $10 \%$ and the amount of di-caffeoylquinic acids was decreased by about $20 \%$. Thus, the proposed extraction method was heating for less than an hour in boiling water, to minimize artifacts in the analyzes of mono- and di-caffeoylquinic acids.

The highest content of flavones and flavonols $(33.78 \mathrm{mg} / 100 \mathrm{~mL})$ was observed in the decoction obtained by boiling the herb of $A$. asiatica for $30 \mathrm{~min}$ and minimal content $(19.01 \mathrm{mg} / 100 \mathrm{~mL})$ was observed for cold infusion. It is interesting, that contents of rhamnosyl-glucosides of quercetin and kaempferol, as well as glucosides of quercetin and apigenin, glucuronides of luteolin and apigenin and kaempferol-3-O-rhamnoside increased with rising extraction temperature. Meanwhile, the content of quercetin-3-O-(6"-O-malonyl)-glucoside and luteolin-3-O-(6"-O-malonyl)-glucoside decreased to trace amounts in samples obtained by boiling infusion and decoctions. This simple phenomenon was described by Katsube et al. where they proved that heat treatment at high temperatures induced the release of $\mathrm{CO}_{2}$ from the malonyl group of quercetin-3-O-(6"-O-malonyl)-glucoside, resulting in the 
formation of quercetin-3-O-(6-O-acetyl)-glucoside [92]. However, quercetin-3-O-(6-O-acetyl)-glucoside was not found in the Agrimonia tea infusions and decoctions in the present study.

The $\alpha$-Glucosidase inhibiting potential of infusions and decoctions obtained was studied. As shown previously, the correlation analysis showed a univocal relationship between the concentration of ellagitannins in the plant extract and its inhibitory activity. So, the A. asiatica herb was characterized by a high content of agrimoniin, and therefore high inhibitory activity against digestive enzyme $\alpha$-glucosidase in dosage forms with the highest expected content of this component. The maximum inhibition of $\alpha$-glucosidase was observed for hot infusion $(75.33 \mu \mathrm{g} / \mathrm{mL})$, and the minimum for $30 \mathrm{~min}$ decoction $(159.14 \mu \mathrm{g} / \mathrm{mL})$. Probably due to the high content of agrimoniin in the hot infusion, this dosage form showed the greatest activity in inhibiting $\alpha$-glucosidase. Thus, the quantitative and qualitative changes of the phenolic profile in Agrimonia tea infusions and decoctions are strongly influenced by heating temperatures. A similar analysis of functional polyphenols in the herb of A. asiatica from this research is useful for standardization and physiological estimation of a perspective functional beverage-Agrimonia herbal tea. To meet the legitimate demands (recommended daily allowance, reference nutritional intake), in vitro investigations should be maintained by in vivo researches to define the bioavailability and bioaccessibility of Agrimonia herbal tea. There is currently a renaissance in food technology that allows the creation of new functional products with health-promoting effects from Conilon and Arabica coffee flowers [93], green tea [94-100], olive [101], Sabah snake grass [102], etc. It is suggested that Agrimonia herbal tea may be part of the regular diet of people with diabetes to improve health or decrease the risk of this illness that goes beyond basic nutritional functions.

\section{Conclusions}

In this paper, a screening investigation of the extracts of 85 Rosaceous tea plants was carried out and Agrimonia asiatica was found to be the extract with the highest inhibitory activity against the $\alpha$-glucosidase enzyme. As a result of chromato-mass-spectrometric profiling of $A$. asiatica herb with high-performance liquid chromatography with photodiode array and electrospray triple quadrupole mass-spectrometric detection, 60 compounds were identified, including catechins, ellagitannins, flavones, flavonols, gallotannins, hydroxycinnamates, procyanidins. To identify the compounds of $A$. asiatica herb with the highest $\alpha$-glucosidase inhibitory activity $A$. asiatica herb extract was subjected to HPLC activity-based profiling which resulted in 5 active fractions. The most pronounced inhibition of $\alpha$-glucosidase was observed for agrimoniin, while less significant results of inhibition were revealed for ellagic acid and isoquercitrin. The comparison of phenolic content in A. asiatica herb infusions and decoctions depending on the method of obtaining herbal tea with the subsequent determination of $\alpha$-glucosidase inhibiting potential was also carried out. It was found that maximum inhibition of $\alpha$-glucosidase was observed for hot infusion and the minimum for $30 \mathrm{~min}$ decoction. This investigation demonstrated that Agrimonia herbal tea is a feasible functional beverage and its dietary intake may help to decrease postprandial hyperglycemia.

Supplementary Materials: The following are available online at http:/www.mdpi.com/2304-8158/9/10/1348/s1, Table S1: Description of 85 Rosaceous plants used in the study, Table S2: Mass spectrometric parameters of compounds 1-60.

Author Contributions: Conceptualization, N.I.K. and D.N.O.; methodology, N.I.K. and D.N.O.; software, N.I.K. and D.N.O.; validation, N.I.K. and D.N.O.; formal analysis, N.I.K. and D.N.O.; investigation, N.I.K. and D.N.O.; resources, N.I.K. and D.N.O.; data curation, N.I.K. and D.N.O.; writing-original draft preparation, N.I.K. and D.N.O.; writing-review and editing, N.I.K. and D.N.O.; visualization, N.I.K. and D.N.O.; supervision, N.I.K. and D.N.O.; project administration, N.I.K.; funding acquisition, N.I.K. and D.N.O. All authors have read and agreed to the published version of the manuscript.

Funding: This research was funded by the Ministry of Education and Science of the Russian Federation, grant numbers AAAA-A17-117011810037-0; FSRG-2020-0019; and Russian Foundation for Basic Research, grant number 18-33-00414.

Acknowledgments: The authors acknowledge the Buryat Research Resource Center for technical support in chromatographic and mass-spectrometric research. 
Conflicts of Interest: The authors declare no conflict of interest. The funders had no role in the design of the study; in the collection, analyses, or interpretation of data; in the writing of the manuscript, or in the decision to publish the results.

\section{References}

1. Galanakis, C.M. The food systems in the era of the Coronavirus (COVID-19) pandemic crisis. Foods 2020, 9, 523. [CrossRef]

2. Galanakis, C.M. Separation of functional macromolecules and micromolecules: From ultrafiltration to the border of nanofiltration. Trends Food Sci. Technol. 2015, 42, 44-63. [CrossRef]

3. Galanakis, C.M. Emerging technologies for the production of nutraceuticalsfrom agricultural by-products: A viewpoint of opportunities and challenges. Food Bioprod. Process. 2013, 91, 575-579. [CrossRef]

4. Tolun, A.; Altintas, Z. Medicinal properties and functional components of beverages. In Functional and Medicinal Beverages; Grumezescu, A.M., Holban, A.M., Eds.; Academic Press: Cambridge, MA, USA, 2019; Volume 11, pp. 235-284.

5. Corbo, M.R.; Bevilacqua, A.; Petruzzi, L.; Casanova, F.P.; Sinigaglia, M. Functional beverages: The emerging side of functional foods commercial trends, research and health implications. Compr. Rev. Food Sci. Food Saf. 2014, 13, 1192-1206. [CrossRef]

6. Chandrasekaraa, A.; Shahidib, F. Herbal beverages: Bioactive compounds and their role in disease risk reduction-A review. J. Tradit. Complement. Med. 2018, 8, 451-458. [CrossRef]

7. Suliburska, J.; Bogdanski, P.; Szulinska, M.; Stepien, M.; Pupek-Musialik, D.; Jablecka, A. Effects of green tea supplementation on elements, total antioxidants, lipids, and glucose values in the serum of obese patients. Biol. Trace Elem. Res. 2012, 149, 315-322. [CrossRef]

8. Tamaya, K.; Matsui, T.; Toshima, A.; Noguchi, M.; Ju, Q.; Miyata, Y.; Tanaka, T.; Tanaka, K. Suppression of blood glucose level by a new fermented tea obtained by tea-rolling processing of loquat (Eriobotrya japonica) and green tea leaves in disaccharide-loaded Sprague-Dawley rats. J. Sci. Food Agric. 2010, 90, 779-783. [CrossRef]

9. Tang, W.; Li, S.; Liu, Y.; Huang, M.-T.; Ho, C.-T. Anti-diabetic activity of chemically profiled green tea and black tea extracts in a type 2 diabetes mice model via different mechanisms. J. Funct. Foods. 2013, 5, 1784-1793. [CrossRef]

10. Hiasa, M.; Kurokawa, M.; Akita, H.; Harada, M.; Niki, K.; Ohta, K.; Shoji, M.; Echigo, N.; Kuzuhara, T. Suppression of increased blood glucose levels in mice by Awa-ban tea following oral administration of monoand disaccharides. J. Funct. Foods 2014, 8, 188-192. [CrossRef]

11. Papatheodorou, K.; Banach, M.; Bekiari, E.; Rizzo, M.; Edmonds, M. Complications of Diabetes 2017. J. Diabetes Res. 2018, 3086167. [CrossRef]

12. Rugerio-Escalona, C.; Ordaz-Pichardo, C.; Becerra-Martinez, E.; Cruz-López, M.D.C.; López-Y-López, V.E.; Mendieta-Moctezuma, A.; Maldonado-Mendoza, I.E.; Jiménez-Montejo, F.E. “Diabetes and metabolism disorders medicinal plants: A glance at the past and a look to the future 2018": Antihyperglycemic activity of Hamelia patens Jacq. Extracts. Evid. Based Complement. Altern. Med. 2018, 7926452. [CrossRef] [PubMed]

13. Lawag, I.L.; Aguinaldo, A.M.; Naheed, S.; Mosihuzzaman, M. $\alpha$-Glucosidase inhibitory activity of selected Philippine plants. J. Ethnopharmacol. 2012, 144, 217-219. [CrossRef] [PubMed]

14. Gao, H.; Huang, Y.; Gao, B.; Kawabata, J. Chebulagic acid is a potent $\alpha$-glucosidase inhibitor. Biosci. Biotechnol. Biochem. 2008, 72, 601-603. [CrossRef] [PubMed]

15. Matsuura, H.; Miyazaki, H.; Asakawa, C.; Amano, M.; Yoshihara, T.; Mizutani, J. Isolation of $\alpha$-glusosidase inhibitors from hyssop (Hyssopus officinalis). Phytochemistry 2004, 65, 91-97. [CrossRef] [PubMed]

16. Kihara, Y.; Ogami, Y.; Tabaru, A.; Unoki, H.; Otsuki, M. Safe and effective treatment of diabetes mellitus associated with chronic liver diseases with an alpha-glucosidase inhibitor, acarbose. J. Gastroenterol. 1997, 32, 777-782. [CrossRef]

17. Asgar, M.A. Anti-diabetic potential of phenolic compounds: A review. Int. J. Food Prop. 2013, 16, 91-103. [CrossRef]

18. Lin, D.; Xiao, M.; Zhao, J.; Li, Z.; Xing, B.; Li, X.; Kong, M.; Li, L.; Zhang, Q.; Liu, Y.; et al. An overview of plant phenolic compounds and their importance in human nutrition and management of type 2 diabetes. Molecules 2016, 21, 1374. [CrossRef] 
19. Landete, J.M. Ellagitannins, ellagic acid and their derived metabolites: A review about source, metabolism, functions and health. Food Res. Int. 2011, 44, 1150-1160. [CrossRef]

20. Bowers, J.J.; Gunawardena, H.P.; Cornu, A.; Narvekar, A.S.; Richieu, A.; Deffieux, D.; Quideau, S.; Tharayil, N. Rapid screening of ellagitannins in natural sources via targeted reporter ion triggered tandem mass spectrometry. Sci. Rep. 2018, 8, 10399. [CrossRef]

21. Han, D.-G.; Cho, S.-S.; Kwak, J.-H.; Yoon, I.-S. Medicinal plants and phytochemicals for diabetes mellitus: Pharmacokinetic characteristics and herb-drug interactions. J. Pharm. Investig. 2019, 49, 603-612. [CrossRef]

22. Olennikov, D.N. Ellagitannins and other phenolic compounds from Comarum palustre. Chem. Nat. Compd. 2016, 52, 721-723. [CrossRef]

23. Olennikov, D.N.; Kruglova, M.Y. A new quercetin glucoside and other phenolic compounds from the genus Filipendula. Chem. Nat. Compd. 2013, 49, 610-616. [CrossRef]

24. Olennikov, D.N.; Kashchenko, N.I.; Chirikova, N.K.; Kuz'mina, S.S. Phenolic profile of Potentilla anserina L. (Rosaceae) herb of Siberian origin and development of a rapid method for simultaneous determination of major phenolics in P. anserina pharmaceutical products by microcolumn RP-HPLC-UV. Molecules 2014, 20, 224-248. [CrossRef]

25. Kashchenko, N.I.; Chirikova, N.K.; Olennikov, D.N. Acylated flavonoids from Spiraea genus as inhibitors of $\alpha$-amylase. Russ. J. Bioorg. Chem. 2018, 44, 876-886. [CrossRef]

26. Olennikov, D.N.; Kashchenko, N.I. New flavonoids and turkesterone-2-O-cinnamate from leaves of Rhaponticum uniflorum. Chem. Nat. Compd. 2019, 55, 256-264. [CrossRef]

27. Olennikov, D.N.; Fedorov, I.A.; Kashchenko, N.I.; Chirikova, N.K.; Vennos, C. Khellactone derivatives and other phenolics of Phlojodicarpus sibiricus (Apiaceae): HPLC-DAD-ESI-QQQ-MS/MS and HPLC-UV profile, and antiobesity potential of dihydrosamidin. Molecules 2019, 24, 2286. [CrossRef]

28. Olennikov, D.N.; Kashchenko, N.I.; Chirikova, N.K. Meadowsweet teas as new functional beverages: Comparative analysis of nutrients, phytochemicals and biological effects of four Filipendula species. Molecules 2017, 22, 16. [CrossRef]

29. Kashchenko, N.I.; Chirikova, N.K.; Olennikov, D.N. Agrimoniin, an active ellagitannin from Comarum palustre herb with anti- $\alpha$-glucosidase and antidiabetic potential in streptozotocin-induced diabetic rats. Molecules 2017, 22, 73. [CrossRef]

30. Gray, A.M.; Flatt, P.R. Actions of the traditional anti-diabetic plant, Agrimony eupatoria (Agrimony): Effects on hyperglycaemia, cellular glucose metabolism and insulin secretion. Br. J. Nutr. 1998, 80, 109-114. [CrossRef]

31. Kuczmannová, A.; Balažová, A.; Račanská, E.; Kameníková, M.; Fialová, S.; Majerník, J.; Nagy, M.; Gál, P.; Mučaji, P. Agrimonia eupatoria L. and Cynara cardunculus L. water infusions: Comparison of anti-diabetic activities. Molecules 2016, 21, 564. [CrossRef]

32. Ibragimov, A.Y.; Khazanovich, R.L. Ursolic acid from Agrimonia asiatica. Chem. Nat. Compd. 1972, 8, 390-391. [CrossRef]

33. Liu, W.-J.; Hou, X.-Q.; Chen, H.; Liang, J.-Y.; Sun, J.-B. Chemical constituents from Agrimonia pilosa Ledeb. and their chemotaxonomic significance. Nat. Prod. Res. 2016, 30, 2495-2499. [CrossRef]

34. Correia, H.; González-Paramás, A.; Amaral, M.T.; Santos-Buelga, C.; Batista, M.T. Polyphenolic profile characterization of Agrimonia eupatoria L. by HPLC with different detection devices. Biomed. Chromatogr. 2006, 20, 88-94. [CrossRef]

35. Pukalskienè, M.; Slapšytè, G.; Dedonytè, V.; Lazutka, J.R.; Mierauskienė, J.; Venskutonis, P.R. Genotoxicity and antioxidant activity of five Agrimonia and Filipendula species plant extracts evaluated by comet and micronucleus assays in human lymphocytes and Ames Salmonella/microsome test. Food Chem. Toxicol. 2018, 113, 303-313. [CrossRef]

36. Granica, S.; Kluge, H.; Horn, G.; Matkowski, A.; Kiss, A.K. The phytochemical investigation of Agrimonia eupatoria L. and Agrimonia procera Wallr. as valid sources of Agrimoniae herba-The pharmacopoeial plant material. J. Pharm. Biomed. Anal. 2015, 114, 272-279. [CrossRef]

37. Nguyen, D.H.; Seo, U.M.; Zhao, B.T.; Min, B.S.; Woo, M.H. Ellagitannin and flavonoid constituents from Agrimonia pilosa Ledeb. with their protein tyrosine phosphatase and acetylcholinesterase inhibitory activities. Bioorg. Chem. 2017, 72, 293-300. [CrossRef]

38. Pei, Y.H.; Li, X.; Zhu, T.R. Studies on the structure of a new ellagic acid glycoside from the root-sprouts of Agrimonia pilosa Ledeb. Acta Pharm. Sin. 1990, 24, 798-800. 
39. Linh, N.V.; Anh, H.L.T.; Yen, D.T.H.; Tai, B.H.; Kiem, P.V. Phenolic components from the aerial parts of Agrimonia pilosa. Nat. Prod. Commun. 2017, 12, 1079-1080. [CrossRef]

40. Jiang, Q.; Ma, J.; Wang, Y.; Chen, L.; Qiu, F. Simultaneous determination of nine major constituents in Agrimonia pilosa Ledeb. by HPLC-DAD-ESI-MS/MS. Anal. Methods 2014, 6, 4373-4379. [CrossRef]

41. Kouno, I.; Baba, N.; Ohni, Y.; Kawano, N. Triterpenoids from Agrimonia pilosa. Phytochemistry 1988, 27, 297-299. [CrossRef]

42. Yamaki, M.; Kashihara, M.; Ishiguro, K.; Takagi, S. Antimicrobial principles of Xian he cao (Agrimonia pilosa). Planta Med. 1989, 55, 169-170. [CrossRef]

43. Tang, L.; Fu, L.; Lu, C.; Shan, W.; Zhan, Z. New cytotoxic phloroglucinol derivatives from Agrimonia pilosa. Fitoterapia 2017, 118, 69-72. [CrossRef] [PubMed]

44. Pei, Y.H.; Li, X.; Zhu, T.R. Studies on the chemical constituents from the root-sprouts of Agrimonia pilosa Ledeb. Acta Pharm. Sin. 1989, 24, 431-437.

45. Granica, S.; Krupa, K.; Klebowska, A.; Kiss, A.K. Development and validation of HPLC-DAD-CAD-MS3 method for qualitative and quantitative standardization of polyphenols in Agrimoniae eupatoriae herba (Ph. Eur). J. Pharm. Biomed. Anal. 2013, 86, 112-122. [CrossRef] [PubMed]

46. Yamato, M. On the chemical structure of agrimonolide, a new constituent of Agrimonia pilosa Ledeb. II. Yakugaku Zasshi 1959, 79, 1069-1073. [CrossRef]

47. Taira, J.; Nanbu, H.; Ueda, K. Nitric oxide-scavenging compounds in Agrimonia pilosa Ledeb on LPS-induced RAW264.7 macrophages. Food Chem. 2009, 115, 1221-1227. [CrossRef]

48. Pei, Y.H.; Li, X.; Zhu, T.R. Studies on the structure of a new isocoumarin glucoside of the root sprouts of Agrimonia pilosa Ledeb. Acta Pharm. Sin. 1989, 24, 837-840.

49. Kim, H.W.; Park, J.; Kang, K.B.; Kim, J.; Sung, S.H. Acylphloroglucinolated catechin and phenylethyl isocoumarin derivatives from Agrimonia pilosa. J. Nat. Prod. 2016, 79, 2376-2383. [CrossRef]

50. Kato, H.; Li, W.; Koike, M.; Wang, Y.; Koike, K. Phenolic glycosides from Agrimonia pilosa. Phytochemistry 2010, 71, 1925-1929. [CrossRef]

51. Drozd, G.A.; Yavlyanskaya, S.F.; Inozemtseva, T.M. Phytochemical investigation of Agrimonia eupatoria. Chem. Nat. Compd. 1983, 19, 104. [CrossRef]

52. Kimura, Y.; Takido, M.; Yamanouchi, S. Studies on the standardization of crude drugs. XI. Constituents of Agrimonia pilosa var. japonica. Yakugaku Zasshi 1968, 88, 1355-1357. [CrossRef] [PubMed]

53. Kim, S.B.; Hwang, S.H.; Suh, H.-W.; Lim, S.S. Phytochemical analysis of Agrimonia pilosa Ledeb, its antioxidant activity and aldose reductase inhibitory potential. Int. J. Mol. Sci. 2017, 18, 379. [CrossRef]

54. Na, B.; Nguyen, P.-H.; Zhao, B.-T.; Min, B.S.; Woo, M.H. Protein tyrosine phosphatase 1B (PTP1B) inhibitory activity and glucosidase inhibitory activity of compounds isolated from Agrimonia pilosa. Pharm. Biol. 2016, 54, 474-480. [CrossRef] [PubMed]

55. Bilia, A.R.; Palme, E.; Marsili, A.; Pistelli, L.; Morelli, I. A flavonol glycoside from Agrimonia eupatoria. Phytochemistry 1993, 32, 1078-1079. [CrossRef]

56. Jung, M.; Park, M. Acetylcholinesterase inhibition by flavonoids from Agrimonia pilosa. Molecules 2007, 12, 2130-2139. [CrossRef] [PubMed]

57. Pan, Y.; Liu, H.-X.; Zhuang, Y.-L.; Chen, L.-X.; Qiu, F. Studies on isolation and identification of flavonoids in herbs of Agrimonia pilosa. Zhongguo Zhongyao Zazhi 2008, 33, 2925-2928.

58. Xu, X.; Qi, X.; Wang, W.; Chen, G. Separation and determination of flavonoids in Agrimonia pilosa Ledeb. by capillary electrophoresis with electrochemical detection. J. Sep. Sci. 2005, 28, 647-652. [CrossRef]

59. Kasai, S.; Watanabe, S.; Kawabata, J.; Tahara, S.; Mizutani, J. Antimicrobial catechin derivatives of Agrimonia pilosa. Phytochemistry 1992, 31, 787-789. [CrossRef]

60. Taira, J.; Ohmine, W.; Ogi, T.; Nanbu, H.; Ueda, K. Suppression of nitric oxide production on LPS/IFN- $\gamma$-stimulated RAW264.7 macrophages by a novel catechin, pilosanol N, from Agrimonia pilosa Ledeb. Bioorg. Med. Chem. Lett. 2012, 22, 1766-1769. [CrossRef]

61. Seo, U.M.; Nguyen, D.H.; Zhao, B.T.; Min, B.S.; Woo, M.H. Flavanonol glucosides from the aerial parts of Agrimonia pilosa Ledeb. and their acetylcholinesterase inhibitory effects. Carbohydr. Res. 2017, 445, 75-79. [CrossRef]

62. Pei, Y.H.; Li, X.; Zhu, T.R.; Wu, L.J. Studies on the structure of a new flavanonol glucoside of the root-sprouts of Agrimonia pilosa Ledeb. Acta Pharm. Sin. 1990, 25, 267-270. 
63. Okuda, T.; Yoshida, T.; Kuwahara, M.; Memon, M.U.; Shingu, T. Agrimoniin and potentillin, an ellagitannin dimer and monomer having an $\alpha$-glucose core. J. Chem. Soc. Chem. Commun. 1982, 163-164. [CrossRef]

64. Okuda, T.; Yoshida, T.; Kuwahara, M.; Memon, U.; Shingu, T. Tannins of Rosaceous medicinal plants. I. Structures of potentillin, agrimonic acids A and B, and agrimoniin, a dimeric ellagitannin. Chem. Pharm. Bull. 1984, 32, 2165-2173. [CrossRef]

65. Tian Zhao, B.; Hung Nguyen, D.; Mi Lee, B.; Sun Min, B.; Hee Woo, M. PTP1B inhibitory and cytotoxic activities of triterpenoids from the aerial parts of Agrimonia pilosa. Med. Chem. Res. 2017, 26, 2870-2878. [CrossRef]

66. An, R.-B.; Kim, H.-C.; Jeong, G.-S.; Oh, H.; Kim, Y.-C. Constituents of the aerial parts of Agrimonia pilosa. Nat. Prod. Sci. 2005, 11, 196-198.

67. Ahn, E.-K.; Lee, J.A.; Seo, D.-W.; Hong, S.S.; Oh, J.S. 1ß-Hydroxy-2-oxopomolic acid isolated from Agrimonia pilosa extract inhibits adipogenesis in 3T3-L1 cells. Biol. Pharm. Bull. 2012, 35, 643-649. [CrossRef]

68. Ma, J.-H.; Jiang, Q.-H.; Chen, Y.; Chen, L.-X.; Feng, Q. A new triterpenoid from the aerial parts of Agrimonia pilosa. Nat. Prod. Commun. 2015, 10, 2041-2044. [CrossRef]

69. Olennikov, D.N.; Chirikova, N.K.; Kashchenko, N.I.; Gornostai, T.G.; Selyutina, I.Y.; Zilfikarov, I.N. Effect of low temperature cultivation on the phytochemical profile and bioactivity of Arctic plants: A case of Dracocephalum palmatum. Int. J. Mol. Sci. 2017, 18, 2579. [CrossRef]

70. Okuda, T.; Yoshida, T.; Hatano, T. Hydrolysable tannins and related polyphenols. Prog. Chem. Org. Nat. Prod. 1995, 66, 1-117. [CrossRef]

71. Hager, J.T.; Howard, L.R.; Liyanage, R.; Lay, J.O.; Prior, R.L. Ellagitannin composition of blackberry as determined by HPLC-ESI-MS and MALDI-TOF-MS. J. Agric. Food Chem. 2008, 56, 661-669. [CrossRef]

72. Lee, J.-H.; Johnson, J.V.; Talcott, S.T. Identification of ellagic acid conjugates and other polyphenolics in muscadine grapes by HPLC-ESI-MS. J. Agric. Food Chem. 2005, 53, 6003-6010. [CrossRef] [PubMed]

73. Mena, P.; Calani, L.; Dall'Asta, C.; Galaverna, G.; Garcia-Viguera, C.; Bruni, R.; Crozier, A.; del Rio, D. Rapid and comprehensive evaluation of (poly)phenolic compounds in pomegranate (Punica granatum L.) juice by UHPLC-MSn. Molecules 2012, 17, 14821-14840. [CrossRef] [PubMed]

74. Regueiro, J.; Sanchez-Gonzalez, C.; Vallverdu-Queralt, A.; Simal-Gandara, J.; Lamuela-Raventos, R.; Izquierdo-Pulido, M. Comprehensive identification of walnut polyphenols by liquid chromatography coupled to linear ion trap-Orbitrap mass spectrometry. Food Chem. 2014, 152, 340-348. [CrossRef] [PubMed]

75. Hatano, T.; Kira, R.; Yoshizaki, M.; Okuda, T. Seasonal changes in the tannins of Liquidambar formosana reflecting their biogenesis. Phytochemistry 1986, 25, 2787-2789. [CrossRef]

76. Okuda, T.; Yoshida, T.; Hatano, T. Hydrolyzable tannins and related polyphenols. In Fortschritte der Chemie organischer Naturstoffe/Progress in the Chemistry of Organic Natural Products; Springer: Wien, Austria, 1995; Volume 66, pp. 83-84.

77. Duckstein, S.M.; Lotter, E.M.; Meyer, U.; Lindequist, U.; Stintzing, F.C. Phenolic constituents from Alchemilla vulgaris L. and Alchemilla mollis (Buser) Rothm. at different dates of harvest. Z. Naturforsch. C 2013, 68, 529-540. [CrossRef]

78. Grochowski, D.M.; Skalicka-Woźniak, K.; Orhan, I.E.; Xiao, J.; Locatelli, M.; Piwowarski, J.P.; Granica, S.; Tomczyk, M. A comprehensive review of agrimoniin. Ann. N. Y. Acad. Sci. 2017, 1401, 166-180. [CrossRef]

79. Tuominen, A.; Salminen, J.-P. Hydrolyzable tannins, flavonol glycosides, and phenolic acids show seasonal and ontogenic variation in Geranium sylvaticum. J. Agric. Food Chem. 2017, 65, 6387-6403. [CrossRef]

80. Salminen, J.-P.; Roslin, T.; Karonen, M.; Sinkkonen, J.; Pihlaja, K.; Pulkkinen, P. Seasonal variation in the content of hydrolyzable tannins, flavonoid glycosides, and proanthocyanidins in oak leaves. J. Chem. Ecol. 2004, 30, 1675-1693. [CrossRef]

81. Salminen, J.-P.; Ossipov, V.; Haukioja, E.; Pihlaja, K. Seasonal variation in the content of hydrolyzable tannins in leaves of Betula pubescens. Phytochemistry 2001, 57, 15-22. [CrossRef]

82. Okuda, T.; Ito, H. Tannins of constant structure in medicinal and food plants-Hydrolyzable tannins and polyphenols related to tannins. Molecules 2011, 16, 2191-2217. [CrossRef]

83. Wolfender, J.-L.; Marti, G.; Thomas, A.; Bertrand, S. Current approaches and challenges for the metabolite profiling of complex natural extracts. J. Chromatogr. A 2015, 1382, 136-164. [CrossRef] [PubMed]

84. Wennberg, T.; Kreander, K.; Lähdevuori, M.; Vuorela, H.; Vuorela, P. Primary screening of natural products using micro fractionation combined with a bioassay. J. Liq. Chromatogr. Relat. Technol. 2004, 27, 2573-2592. [CrossRef] 
85. Toda, M.; Kawabata, J.; Kasai, T. Inhibitory effects of ellagi- and gallotannins on rat intestinal $\alpha$-glucosidase complexes. Biosci. Biotechnol. Biochem. 2001, 65, 542-547. [CrossRef] [PubMed]

86. Spencer, C.M.; Cai, Y.; Martin, R.; Gaffney, S.H.; Goulding, P.N.; Mangnolato, D.; Lilley, Y.; Haslam, E. Polyphenol complexation-Some thoughts and observatios. Phytochemistry 1988, 27, 2397-2409. [CrossRef]

87. David, B.; Wolfender, J.-L.; Dias, D. The pharmaceutical industry and natural products: Historical status and new trends. Phytochem. Rev. 2014, 14, 299-315. [CrossRef]

88. Sõukand, R.; Quave, C.L.; Pieroni, A.; Pardo-de-Santayana, M.; Tardío, J.; Kalle, R.; Łuczaj, Ł.; Svanberg, I.; Kolosova, V.; Aceituno-Mata, L.; et al. Plants used for making recreational tea in Europe: A review based on specific research sites. J. Ethnobiol. Ethnomed. 2013, 9, 58. [CrossRef] [PubMed]

89. Martins, N.; Barros, L.; Santos-Buelga, C.; Silva, S.; Henriques, M.; Ferreira, I.C. Decoction, infusion and hydroalcoholic extract of cultivated thyme: Antioxidant and antibacterial activities, and phenolic characterization. Food Chem. 2015, 167, 131-137. [CrossRef] [PubMed]

90. Jordao, A.M.; Ricardo-da-Silva, J.M.; Laureano, O. Extraction of some ellagic tannins and ellagic acid from oak wood chips (Quercus pyrenaica L.) in model wine solutions: Effect of time, $\mathrm{pH}$, temperature and alcoholic content. S. Afr. J. Enol. Viticult. 2005, 26, 83-89. [CrossRef]

91. Li, Y.-J.; Zhang, C.-F.; Ding, G.; Huang, W.-Z.; Wang, Z.-Z.; Bi, Y.-A.; Xiao, W. Investigating the thermal stability of six caffeoylquinic acids employing rapid-resolution liquid chromatography with quadrupole time-of-flight tandem mass spectrometry. Eur. Food Res. Technol. 2015, 240, 1225-1234. [CrossRef]

92. Katsube, T.; Tsurunaga, Y.; Sugiyama, M.; Furuno, T.; Yamasaki, Y. Effect of air-drying temperature on antioxidant capacity and stability of polyphenolic compounds in mulberry (Morus alba L.) leaves. Food Chem. 2009, 113, 964-969. [CrossRef]

93. De Abreu Pinheiro, F.; Elias, L.F.; de Jesus Filho, M.; Modolo, M.U.; Rocha, J.D.C.G.; Lemos, M.F.; Scherer, R.; Cardoso, W.S. Arabica and Conilon coffee flowers: Bioactive compounds and antioxidant capacity under different processes. Food Chem. 2020, 336, 127701. [CrossRef] [PubMed]

94. Pakfetrat, S.; Amiri, S.; Radi, M.; Abedi, E.; Torri, L. The influence of green tea extract as the steeping solution on nutritional and microbial characteristics of germinated wheat. Food Chem. 2020, 332, 127288. [CrossRef] [PubMed]

95. Yoo, S.-H.; Lee, Y.-E.; Chung, J.-O.; Rha, C.-S.; Hong, Y.-D.; Park, M.-Y.; Shim, S.-M. Enhancing the effect of catechins with green tea flavonol and polysaccharides on preventing lipid absorption and accumulation. LWT 2020, 134, 110032. [CrossRef]

96. Akhtar, N.; Thadhani, V.M.; Haq, F.U.; Khan, M.N.; Ali, S.; Musharraf, S.G. Rapid identification and quantification of bioactive metabolites in processed Camellia sinensis samples by UHPLC-ESI-MS/MS and evaluation of their antioxidant activity. J. Ind. Eng. Chem. 2020, 90, 419-426. [CrossRef]

97. Liu, Y.; Zhao, G.; Li, X.; Shen, Q.; Wu, Q.; Zhuang, J.; Zhang, X.; Xia, E.; Zhang, Z.; Qian, Y.; et al. Comparative analysis of phenolic compound metabolism among tea plants in the section Thea of the genus Camellia. Food Res. Int. 2020, 135, 109276. [CrossRef]

98. Reddy, C.K.; Jung, E.S.; Son, S.Y.; Lee, C.H. Inclusion complexation of catechins-rich green tea extract by $\beta$-cyclodextrin: Preparation, physicochemical, thermal, and antioxidant properties. LWT 2020, 131, 109723. [CrossRef]

99. Wang, J.-Q.; Hu, X.-F.; Du, Q.-Z.; Zeng, L.; Wang, S.; Yin, J.-F.; Xu, Y.-Q. Effect of tannase on sediment formation in green tea infusion. J. Food Meas. Charact. 2020, 14, 1957-1965. [CrossRef]

100. Sentkowska, A.; Piwowarczyk, S.; Pyrzyńska, K. Simultaneous determination of vitamin B6 and catechins in dietary supplements by ZIC-HILIC chromatography and their antioxidant interactions. Eur. Food Res. Technol. 2020, 246, 1609-1615. [CrossRef]

101. Galanakis, C.M.; Tsatalas, P.; Galanakis, I.M. Implementation of phenols recovered from olive mill wastewater as UV booster in cosmetics. Ind. Crop. Prod. 2018, 111, 30-37. [CrossRef]

102. Shafie, N.S.M.; Latif, M.S.; Ghazali, M.I.; Abbas, S.; Kormin, F. Microwave-assisted extraction optimization of sabah snake grass (Clinacanthus nutans lindau) herbal infusion and evaluation of antioxidant and acetylcholinesterase inhibition activity in vitro. Food Res. 2020, 4, 1710-1718. [CrossRef]

(C) 2020 by the authors. Licensee MDPI, Basel, Switzerland. This article is an open access article distributed under the terms and conditions of the Creative Commons Attribution (CC BY) license (http://creativecommons.org/licenses/by/4.0/). 